\title{
Artigo Original / Original Paper \\ Estágio sucessional de uma floresta estacional semidecidual secundária com distintos históricos de uso do solo no sul do Espírito Santo
}

Successional stage of a semideciduous seasonal secondary forest with different land use history in the southern of Espírito Santo

\author{
Luciana de Souza Lorenzoni-Paschoa ${ }^{1,7}$, Karla Maria Pedra de Abreu ${ }^{2}$, Gilson Fernandes da Silva ${ }^{3}$, \\ Henrique Machado Dias ${ }^{4}$, Luciana Assis Machado ${ }^{5} \&$ Renata de Deus Silva $^{6}$
}

\begin{abstract}
Resumo
Estudos sobre a estrutura vegetacional de fragmentos florestais que consideram o uso pretérito do solo podem contribuir para subsidiar ações conservacionistas e de restauração florestal. A partir disso, objetivamos comparar três sítios de um fragmento florestal com distintas histórias de perturbação e verificar se os mesmos diferem entre si com relação ao estágio sucessional analisando atributos estruturais da vegetação arbórea e abundância de lianas. A área estudada localiza-se no município de Alegre-ES, em uma Floresta Estacional Semidecidual constituída por três sítios com diferentes históricos de uso do solo e idades de pousio que recuperaram-se de corte seletivo (CS), lavoura de café (CC) e pastagem (PA). Utilizamos parcelas (19) de área fixa $(20 \times 20 \mathrm{~m})$ na amostragem, totalizando 0,76 ha. Os indivíduos arbóreos com DAP $\geq 5 \mathrm{~cm}$ e as lianas com DAP $\geq 1 \mathrm{~cm}$ foram amostrados. A análise da categoria sucessional demonstrou que os sítios CS e PA possuem estágio mais avançado de sucessão em relação à CC. A espécie de maior VI, síndromes de dispersão e lianas apontaram maior avanço sucessional do sítio PA, área com maior proteção aos efeitos de borda e antrópicos por estar localizada no interior do fragmento florestal. As demais análises não demonstraram diferença entre os três sítios.
\end{abstract}

Palavras-chave: fitossociologia, conservação, Mata Atlântica.

\begin{abstract}
To consider past land use on vegetative structure studies of forest fragments can contribute to conservation and forest restoration actions. From this we aim to compare three sites of a forest fragment with distinct anthropogenic disturbances and to verify if they differ on successional stage through analyses of structural traits (trees) and non-trees abundance (lianas) The studied area is located in the Alegre municipality, ES state, in a semideciduous seasonal forest matrix with different land use history composed of selective cutting $(\mathrm{CS})$, coffee crop (CC) and pasture (PA). At each site, randomly plots were sample (19 total plots, $20 \times 20$ $\mathrm{m}$ each), totaling 0.76 ha. All tree individuals with $\mathrm{DBH} \geq 5 \mathrm{~cm}$ and liana with $\mathrm{DBH} \geq 1 \mathrm{~cm}$ were sampled. The successional category showed an advanced stage of succession in the CS and PA sites. The specie with higher VI, the dispersion syndromes and the abundance of lianas showed successional advanced stage only in the PA site, because is located inside the forest fragment providing greater protection from edge effects, and anthropogenic disturbances. The others analyses didn't showed statistical differences between the three sites. Key words: phytosociology, conservation, Atlantic Forest.
\end{abstract}

\footnotetext{
1,3,4 Universidade Federal do Espírito Santo (UFES), Depto. Ciências Florestais e da Madeira, Av. Governador Lindemberg 316, 29550-000, Jerônimo Monteiro, ES, Brasil.

${ }^{2,5,6}$ Instituto Federal de Educação, Ciência e Tecnologia do Espírito Santo (Ifes), Campus de Alegre, Rod. 482, km 47, 29520-000, Alegre, ES, Brasil.

${ }^{7}$ Autor para correspondência: lucianaloren27@gmail.com
} 


\section{Introdução}

A Mata Atlântica constitui-se em uma das maiores florestas tropicais do planeta, estendendo-se por vários estados brasileiros, sendo considerada um dos hotspots mundiais para a conservação (Myers et al. 2000). Estima-se que aproximadamente $10,5 \%$ da área do estado do Espírito Santo seja coberto por remanescentes florestais. Dentre esses, a Floreta Estacional Semidecidual (FES) é considerada a segunda mais relevante formação vegetacional em termos de área ocupada (Fundação SOS Mata Atlântica \& INPE 2015).

Na região sul do estado, a ocupação do solo apresenta maior proporção de atividades como pastagens, cafeicultura, silvicultura e mineração. Dentre essas atividades, o corte seletivo para exploração de madeiras nobres, comumente realizado no passado, propiciou a abertura de clareiras, que interferiu no estágio sucessional, aumentando a ocorrência de espécies de início de sucessão em detrimento das secundárias tardias (Carvalho \& Nascimento 2009). Outras atividades mais impactantes como a implantação de pastagens e cafezais, as quais envolvem uma série de modificações do meio, como a remoção total da cobertura florestal, implantação de espécies exóticas como as gramíneas e Coffea sp., à exaustão do banco de sementes e profundas mudanças químicas e físicas no solo (D'Antonio \& Vitorusek 1992).

Devido à exaustiva e insustentável atividade agropecuária na região sul do ES, a área coberta por florestas de Mata Atlântica foi drasticamente reduzida e/ou impactada, o que resultou em pequenos fragmentos perturbados e isolados (Lira et al. 2012), formando uma paisagem de ilhas de vegetação. Nesse contexto, ecólogos tem voltado sua atenção para esses "novos ecossistemas" com o intuito de conservar a biodiversidade remanescente (Oliveira-Neto et al. 2017) em florestas que estão se recuperando por meio de sucessão ecológica. Atualmente, os fragmentos florestais de Mata Atlântica encontram-se em diferentes estágios sucessionais (Lira et al. 2012) e sob diferentes condições de resiliência (Tabarelli et al. 2012), as quais dependem do tempo de pousio, histórico de perturbação e tipo de uso anterior do solo (Marques et al. 2014). O estágio sucessional de uma floresta pode ser conhecido por meio da análise florística e fitossociológica de espécies arbóreas. Além dessas, a presença de lianas também pode ser indicadora de estágio sucessional, pois as mesmas tendem a ser mais abundantes em locais alterados ou de sucessão inicial (Carvalho et al. 2011).

Comparar a estrutura, atributos funcionais de uma comunidade vegetal que passou por diferentes tipos de distúrbios antrópicos, conforme realizado na presente pesquisa, pode fornecer relevantes informações sobre a influência desses na vegetação e em suas trajetórias sucessionais, as quais ainda são incertas para fragmentos florestais impactados (Marques et al. 2014). Essas informações podem subsidiar estratégias de manejo, conservação da biodiversidade e de restauração para outras áreas degradadas (Mota et al. 2017). Como medidas de manejo pode-se indicar plantio de enriquecimento com espécies que compõem a comunidade, aumentando a probabilidade de sucesso (Mota et al. 2017).

Nesse contexto, a presente pesquisa foi desenvolvida em um fragmento de FES composto por três sítios com distintos tempos de pousio e históricos de uso do solo e teve por objetivo comparar esses sítios buscando verificar se os mesmos diferem entre si em termos florísticos e estruturais da vegetação arbórea e abundância de lianas, bem como de sucessão ecológica. Tratamos como hipótese central a ideia de que o sítio corte seletivo (CS) possui características estruturais e florísticas característicos de estágios mais avançados de sucessão e menor abundância de lianas de menor calibre por esta ser uma área que não sofreu corte raso. Acreditamos que o sítio cultivo de café (CC) apresente menor avanço sucessional em relação ao sítio CS, pois, apesar de possuir maior tempo de pousio, regenerou-se a partir de cafezais. Esperamos que o sítio pastagens (PA) tenha atributos florísticos e estruturais, bem como densidade de lianas que indicam sucessão ecológica menos avançada com relação aos demais sítios, por ser a área com menor tempo de pousio e proveniente de uma pastagem abandonada.

\section{Material e Métodos}

Caracterização da área de estudo

O presente estudo foi desenvolvido em um fragmento florestal $(109,6 \mathrm{ha})$ localizado na sub-bacia do Córrego Horizonte, distrito de Rive, município de Alegre, sul do estado do Espírito Santo (20 44'05”S e $\left.41^{\circ} 25^{\prime} 50^{\prime \prime} \mathrm{W}\right)$ (Fig. 1). Segundo a classificação de Köppen (1948), o clima da região é do tipo "Cwa", tropical quente úmido com inverno frio e seco. O município de Alegre 


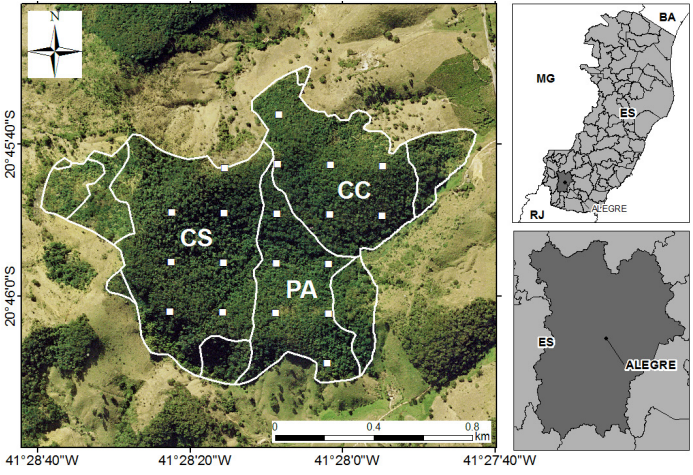

Figura 1 - Localização do Fragmento florestal, município de Alegre-ES, dos sítios e parcelas. $(\mathrm{CS}=$ sítio de corte seletivo; $\mathrm{CC}=$ sítio de cultivo de café; $\mathrm{PA}=$ sítio de pastagens).

Figure 1 - Location of the forest fragment, Alegre-ES municipality, sites and plots. $(\mathrm{CS}=$ selective cutting site; $\mathrm{CC}=$ coffee growing site; $\mathrm{PA}=$ pasture site).

apresenta uma precipitação média anual de 1.341 mm (Lima et al. 2008) e temperatura média anual de 23,1 ${ }^{\circ} \mathrm{C}$ (Pezzopane et al. 2004).

O solo é classificado como Latossolo Vermelho Amarelo (EMBRAPA 2006). A vegetação da região é classificada como Floresta Estacional Semidecidual Submontana (Veloso et al. 1991) e a altitude do fragmento florestal varia de $120 \mathrm{~m}$ a $660 \mathrm{~m}$.

O fragmento florestal estudado é composto pela Reserva Legal do Polo de Educação Ambiental da Mata Atlântica do Ifes Campus de Alegre (PEAMA) e por fazendas circunvizinhas. Sua área total é constituída por três sítios, os quais diferem entre si em relação ao tempo em que foram destinados a pousio e ao tipo de uso anterior do solo. Existem áreas do fragmento florestal, além dos sítios, que totalizam 22,55 ha, que não foram considerados no presente estudo por serem compostas por penhascos e áreas separadas por rochas. O histórico dos sítios foi fornecido, por meio de conversa informal, pelo gestor do PEAMA e por dois funcionários que trabalharam no corte seletivo de madeira. O sítio CS $(39,47$ ha) sofreu corte seletivo, apresentando 47 anos de processo de recuperação, compõe uma das bordas do fragmento e está situado em uma vertente inclinada, a leste. O sítio CC (26,95 ha) continha cafezais, possui aproximadamente 57 anos de regeneração, compõe a outra borda do fragmento e está situada nas mesmas condições de relevo, a sudeste. O sítio PA (20,63 ha) era coberto por pastagem, possui cerca de 43 anos de regeneração e é a maior área situada no interior do fragmento, com grande parte em relevo acidentado, ao norte dos demais sítios. Todos os sítios regeneraram-se naturalmente.

\section{Amostragem}

A amostragem baseou-se em parcelas de área fixa (Mueller-Dombois \& Ellemberg 2002) com $20 \times 20 \mathrm{~m}\left(400 \mathrm{~m}^{2}\right)$ distribuídas em um grid organizado de forma sistemática sobre o fragmento florestal. As parcelas estão 200 metros distantes entre si em todas as quatro direções cardeais. Foram alocadas 19 parcelas no fragmento, sendo sete parcelas $(0,28$ ha) no sítio $\mathrm{CS}$, seis parcelas $(0,24$ ha) no sítio $\mathrm{CC}$ e outras seis parcelas $(0,24$ ha) no sítio PA, resultando em uma área amostral de 0,76 ha.

Foram medidos todos os DAPs (diâmetro a altura de 1,30 metros a partir do nível do solo) de árvores com diâmetro igual ou superior a $5 \mathrm{~cm}$. Os indivíduos foram identificados com plaquetas numeradas e presas aos fustes. Nos casos de indivíduos perfilhados, as ramificações também foram consideradas, desde que estivessem vivas, sendo contabilizados como um indivíduo para cálculo de área basal.

Além das espécies arbóreas, os indivíduos de lianas enraizados em cada parcela e com DAP $\geq 1 \mathrm{~cm}$ foram contabilizados e tiveram os caules mensurados (Carvalho et al. 2011). As lianas não foram coletadas e identificadas, apenas sua abundância e distribuições diamétricas foram consideradas nesse estudo.

\section{Análises dos dados}

A identificação das espécies foi realizada por meio de comparação com as coleções dos Herbários VIES (subcuradoria Jerônimo Monteiro, Departamento de Ciências Florestais e da Madeira do Centro de Ciências Agrárias e Engenharias da Universidade Federal do Espírito Santo) e RB (Instituto de Pesquisas do Jardim Botânico do Rio de Janeiro), além de consultas a especialistas e/ou bibliografia especializada. O material testemunho coletado foi herborizado e depositado no herbário VIES. O sistema de classificação APG IV (2016) foi utilizado para circunscrição das espécies nas respectivas famílias botânicas. A confirmação dos nomes científicos e dos respectivos autores foi embasada na lista da Flora do Brasil (Forzza et al. 2012).

Classificamos as espécies arbóreas em grupos ecológicos como pioneiras (Pi), secundárias iniciais (SI) e secundárias tardias (ST) conforme Gandolfi 
et al. (1995) e quanto às síndromes de dispersão em zoocóricas (Zoo), anemocóricas (Ane) e autocóricas (Aut) (Van der Pijl 1982). Algumas espécies não foram classificadas por carência de informações pertinentes (NC). As espécies consideradas ameaçadas de extinção foram listadas com base no The Red List of Threatened Species (IUCN 2016), na Lista da Flora Brasileira (Martinelli \& Moraes 2013) e na lista do Instituto Estadual de Meio Ambiente -IEMA (Decreto $N^{\circ}$ 1499-R, de 13 de Junho de 2005).

Foram calculados, para os três sítios, os parâmetros fitossociológicos da comunidade arbórea amostrada (Mueller-Dombois \& Ellenberg 2002): valor de importância (calculado com base nos parâmetros relativos de densidade, dominância, frequência e valor de cobertura) e área basal por meio do software Mata Nativa 3 (CIENTEC 2010). Os valores de área basal de espécies arbóreas foram comparados entre os sítios por meio da análise de variância (ANOVA) $(\mathrm{p}<0,05 \%)$, sendo a normalidade dos dados brutos previamente testada. No caso de ocorrência de diferenças estatísticas entre as médias, foi aplicado, a posteriori, o teste de Tukey para discriminar as diferenças ( $\mathrm{p}<0,05 \%)$. Para os dados que não atenderam aos requisitos de um teste paramétrico, foi aplicado o teste não paramétrico de Kruskal-Wallis e, no caso de ocorrência de diferenças estatísticas, foi aplicado, a posteriori, o teste de Dunn. Tais análises foram realizadas com auxílio do software ASSISTAT (Silva \& Azevedo 2009).

Para calcular a similaridade florística entre os sítios foram utilizados os índices qualitativo de similaridade de Sørensen (Kent \& Coker 1992) e o quantitativo de Morisita Horn (Magurran 1988). Foi realizada uma classificação aglomerativa por UPGMA (Unweighted Pair Groups Method using Arithmetic Averages) (James \& McCulloch 1990; Belbin \& McDonald 1993) para elaboração dos dendrogramas de similaridade utilizando o programa MVSP (Kovach 2004).

$\mathrm{O}$ índice de diversidade de Shannon-Weaver (H') e o índice de equabilidade de Pielou (J) (Magurran 2004) foram calculados com auxílio do software Mata Nativa 3 (CIENTEC 2010). A comparação da riqueza de espécies e diversidade foram feitas por meio de curvas de rarefação baseadas em indivíduos, com intervalos de $95 \%$ de confiança, utilizando o programa EcoSim 7 (Gotelli \& Entsminger 2011).

Para a análise da estrutura diamétrica das espécies arbóreas, adotamos uma amplitude de classe de cinco centímetros, conforme Archanjo et al. (2012) e França \& Stehmann (2013), sendo o primeiro centro de classe estabelecido em 7,5 cm. Foi aplicado o teste Qui-Quadrado (Zar 1999) para comparar a densidade de indivíduos em todas as classes de diâmetro existentes entre os três sítios, em nível de significância de 5\%. Os diâmetros das lianas foram contabilizadas e seus valores relativos foram divididos em três classes de diâmetro: classe $1(1 \leq \mathrm{DAP}<2,5 \mathrm{~cm})$, classe $2(2,5 \leq \mathrm{DAP}<5 \mathrm{~cm})$ e classe $3(\mathrm{DAP} \geq 5 \mathrm{~cm})$. Os valores relativos das lianas foram comparados por meio da análise de variância (ANOVA) $(\mathrm{p}<0,05 \%)$ e, quando os dados demonstraram normalidade e diferenças estatísticas entre as médias, foi aplicado, a posteriori, o teste de Tukey ( $\mathrm{p}<0,05 \%)$.

\section{Resultados e Discussão}

Foram registrados 1.347 indivíduos arbóreos no fragmento florestal, sendo 152 espécies pertencentes a 33 famílias. Cerca de $88 \%$ dos indivíduos $(\mathrm{n}=1.188)$ foram identificados em nível de espécie, $7 \%(\mathrm{n}=94)$ em nível de gênero, $4 \%(n=14)$ em nível de família e $1 \%(n=13)$ dos indivíduos não foram identificados (Tab. 1).

Considerando os três sítios em conjunto, as famílias mais ricas foram: Fabaceae (43), Meliaceae (12), Euphorbiaceae (8) e Salicaceae (6), representando $45,4 \%$ das espécies e $76 \%$ dos indivíduos. Os destaques dessas famílias corroboram com outros trabalhos desenvolvidos em Florestas Estacionais Semideciduais (FES) (Braga et al. 2011; Archanjo et al. 2012; Abreu et al. 2013; Estevan et al. 2016). As famílias Fabaceae e Meliaceae apresentaram maior riqueza nos três sítios. Fabaceae quase sempre apresenta alta riqueza florística em FES sendo característica desse tipo de formação vegetal (Braga et al. 2011). Meliaceae, além de apresentar alta riqueza na amostragem, também contribuiu com grande abundância (10\%) indicando que o fragmento em estudo está se recuperando de seu histórico de uso do solo. Meliaceae é caraterística de ambientes menos impactados e sua riqueza tende a aumentar proporcionalmente ao estágio sucessional da floresta (Braga et al. 2011). Além disso, a maioria das espécies dessa família, conforme registrado na presente pesquisa, é zoocórica, contribuindo para a manutenção da diversidade da fauna local.

O sítio CS apresentou 90 espécies, o sítio CC, 72 espécies e o sítio PA, 93 espécies (Tab. 1). Foi verificada a presença de 55 espécies ocorridas com um único indivíduo na amostragem total, as 
Tabela 1 - Lista de espécies arbóreas amostradas em três sítios que compõem o Fragmento florestal, município de Alegre-ES - VI = valor de importância; $\mathrm{Col}=$ colocação das espécies em função do VI; CS = sítio de corte seletivo; $\mathrm{CC}=$ sítio de cultivo de café; $\mathrm{PA}=$ sítio de pastagens; $\mathrm{CSu}=$ categoria sucessional; $\mathrm{Pi}=$ pioneira; $\mathrm{SI}=$ secundáia inicial; $\mathrm{ST}=$ secundaria tardia; $\mathrm{SD}=$ síndrome de dispersão; Ane = anemocórica; Zoo = zoocórica; Aut = autocórica; $\mathrm{NC}=$ não classificada; $\mathrm{CN}=$ número de coletor; $\mathrm{LSL}=$ Luciana de Souza Lorenzoni.

Table 1 - List of tree species sampled on three sites that make up the Forest Fragment, municipality of Alegre-ES - VI $=$ value of importance; $\mathrm{Col}=$ species placement as a function of $\mathrm{LV} ; \mathrm{CS}=$ selective cutting site; $\mathrm{CC}=$ coffee growing site; $\mathrm{PA}=$ pasture site; $\mathrm{CSu}=$ successional category; $\mathrm{Pi}=$ pioneer; $\mathrm{SI}=$ early secondary; $\mathrm{ST}=$ late secondary; $\mathrm{SD}=$ dispersion syndrome; Ane = anemocoric; $\mathrm{Zoo}=$ zoochoric Aut $=$ autocoric $; \mathrm{NC}=$ unclassified $\mathrm{CN}=$ collector number; $\mathrm{LSL}=$ Luciana de Souza Lorenzoni.

\begin{tabular}{|c|c|c|c|c|c|c|c|c|c|}
\hline \multirow{3}{*}{$\begin{array}{l}\text { Família / Espécie } \\
\text { ACHARIACEAE }\end{array}$} & \multicolumn{6}{|c|}{ Sítios } & \multirow{3}{*}{ CSu } & \multirow{3}{*}{ SD } & \multirow{3}{*}{$\mathbf{C N}$} \\
\hline & \multicolumn{2}{|c|}{$\mathrm{CS}$} & \multicolumn{2}{|c|}{$\mathrm{CC}$} & \multicolumn{2}{|c|}{ PA } & & & \\
\hline & VI & Col & VI & Col & VI & Col & & & \\
\hline Carpotroche brasiliensis (Raddi) A Gray & 0,95 & 65 & 5,84 & 12 & - & - & ST & Zoo & LSL et al. 120 \\
\hline \multicolumn{10}{|l|}{ ANACARDIACEAE } \\
\hline Astronium concinnum Schott & 15,19 & 2 & 9,58 & 8 & 7,91 & 9 & ST & Ane & LSL et al. 138 \\
\hline Astronium graveolens Jacq. & 11,09 & 7 & 7,48 & 10 & 6,14 & 12 & SI & Ane & LSL et al. 18 \\
\hline \multicolumn{10}{|l|}{ ANNONACEAE } \\
\hline Annona crassiflora Mart & 1,25 & 57 & - & - & - & - & $\mathrm{Pi}$ & Zoo & LSL et al. 149 \\
\hline Ephedranthus sp. & 1,83 & 43 & 1,37 & 39 & - & - & $\mathrm{NC}$ & $\mathrm{NC}$ & LSL et al. 152 \\
\hline Oxandra nitida Refr. & - & - & 4,02 & 17 & 1,77 & 47 & $\mathrm{NC}$ & Zoo & LSL et al. 16 \\
\hline Xylopia laevigata (Mart.) Refr. & - & - & 0,97 & 64 & - & - & ST & Zoo & LSL et al. 17 \\
\hline \multicolumn{10}{|l|}{ APOCYNACEAE } \\
\hline Aspidosperma sp. & - & - & - & - & 2,27 & 37 & $\mathrm{NC}$ & $\mathrm{NC}$ & LSL et al. 136 \\
\hline Tabernaemontana laeta Mart. & - & - & 1,04 & 52 & - & - & $\mathrm{Pi}$ & Zoo & LSL et al. 151 \\
\hline \multicolumn{10}{|l|}{ BIGNONIACEAE } \\
\hline Adenocalymna sp. & 0,81 & 85 & - & - & - & - & $\mathrm{NC}$ & $\mathrm{NC}$ & LSL et al. 147 \\
\hline Paratecoma peroba (Record) Kuhlm. & 2,17 & 38 & 1,44 & 38 & 1,31 & 55 & $\mathrm{ST}$ & Ane & LSL et al. 150 \\
\hline Tabebuia chrysotricha var. obtusata (DC.) Toledo & - & - & 1,06 & 51 & 7,47 & 11 & ST & Ane & LSL et al. 15 \\
\hline Tabebuia roseoalba (Ridl.) Sandwith & 3,71 & 25 & 2,11 & 33 & 7,89 & 10 & $\mathrm{Si}$ & Ane & LSL et al. 137 \\
\hline \multicolumn{10}{|l|}{ BURSERACEAE } \\
\hline Protium heptaphyllum (Aubl.) Marchand & - & - & - & - & 1,08 & 61 & SI & Zoo & LSL et al. 148 \\
\hline \multicolumn{10}{|l|}{ CELASTRACEAE } \\
\hline Maytenus glaucescens Reissek & - & - & - & - & 0,92 & 78 & $\mathrm{NC}$ & $\mathrm{NC}$ & LSL et al. 119 \\
\hline Maytenus sp. & 3,20 & 29 & - & - & 0,88 & 89 & $\mathrm{NC}$ & $\mathrm{NC}$ & LSL et al. 139 \\
\hline \multicolumn{10}{|l|}{ CHRYSOBALANACEAE } \\
\hline Licania kunthiana Hook.f. & 0,82 & 77 & - & - & - & - & $\mathrm{NC}$ & $\mathrm{NC}$ & LSL et al. 19 \\
\hline \multicolumn{10}{|l|}{ CLUSIACEAE } \\
\hline Kielmeyera excelsa Cambess. & - & - & 1,04 & 53 & 2,12 & 40 & $\mathrm{NC}$ & $\mathrm{NC}$ & LSL et al. 146 \\
\hline \multicolumn{10}{|l|}{ COMBRETACEAE } \\
\hline Terminalia cf. mameluco Pickel & 5,54 & 14 & - & - & 1,15 & 57 & $\mathrm{Si}$ & Zoo & LSL et al. 121 \\
\hline \multicolumn{10}{|l|}{ ERYTHROXYLACEAE } \\
\hline Erythroxylum deciduum A.St.-Hil. & 1,07 & 62 & - & - & - & - & $\mathrm{Si}$ & Zoo & LSL et al. 13 \\
\hline Erythroxylum pulchrum A.St.-Hil. & 1,71 & 45 & 18,12 & 5 & 2,68 & 30 & $\mathrm{ST}$ & Zoo & LSL et al. 14 \\
\hline
\end{tabular}




\begin{tabular}{|c|c|c|c|c|c|c|c|c|c|}
\hline \multirow{2}{*}{$\begin{array}{l}\text { Família / Espécie } \\
\text { EUPHORBIACEAE }\end{array}$} & \multicolumn{6}{|c|}{ Sítios } & \multirow{2}{*}{$\mathrm{CSu}$} & \multirow{2}{*}{ SD } & \multirow{2}{*}{$\mathbf{C N}$} \\
\hline & \multicolumn{2}{|c|}{ CS } & \multicolumn{2}{|c|}{ CC } & \multicolumn{2}{|c|}{ PA } & & & \\
\hline Actinostemon concolor (Spreng.) Müll.Arg. & 11,73 & 5 & 0,97 & 68 & 0,92 & 76 & ST & Aut & LSL et al. 12 \\
\hline Actinostemon verticillatus (Klotzsch) Baill. & 14,86 & 3 & 2,31 & 28 & - & - & ST & Aut & LSL et al. 20 \\
\hline Cnidoscolus oligandra (Müll.Arg.) Pax & 11,33 & 6 & - & - & 2,93 & 26 & $\mathrm{Pi}$ & Aut & LSL et al. 10 \\
\hline Euphorbiaceae sp. & 3,52 & 28 & 2,81 & 22 & 4,62 & 17 & $\mathrm{NC}$ & $\mathrm{NC}$ & LSL et al. 22 \\
\hline Gymnanthes nervosa Müll.Arg. & - & - & 0,98 & 62 & 0,88 & 90 & $\mathrm{NC}$ & $\mathrm{NC}$ & LSL et al. 21 \\
\hline Pachystroma longifolium (Nees) IMJohnst. & 12,16 & 4 & - & - & 1,43 & 52 & SI & Aut & LSL et al. 28 \\
\hline Paradrypetes ilicifolia Kuhlm & 0,98 & 64 & - & - & - & - & $\mathrm{NC}$ & $\mathrm{NC}$ & LSL et al. 26 \\
\hline Sapium sp. & - & - & - & - & 1,09 & 60 & $\mathrm{NC}$ & $\mathrm{NC}$ & LSL et al. 24 \\
\hline Senefeldera verticillata (Vell.) Croizat & 7,41 & 11 & - & - & - & - & ST & Aut & LSL et al. 11 \\
\hline FABACEAE & & & & & & & & & \\
\hline Acosmium lentiscifolium Schott & 3,64 & 26 & 3,30 & 19 & 4,28 & 19 & ST & Ane & LSL et al. 2 \\
\hline Anadenanthera colubrina (Vell.) Brenan & 0,81 & 81 & - & - & 0,89 & 83 & SI & Ane & LSL et al. 23 \\
\hline Amburana cearensis (Allemão) ACSm. & 4,94 & 19 & - & - & 3,26 & 24 & $\mathrm{Pi}$ & Zoo & LSL et al. 27 \\
\hline Andira fraxinifolia Benth. & 1,67 & 46 & - & - & - & - & $\mathrm{Pi}$ & Zoo & LSL et al. 32 \\
\hline Apuleia leiocarpa (Vogel) JFMacbr. & 7,24 & 12 & 35,30 & 1 & 5,96 & 13 & SI & Ane & LSL et al. 1 \\
\hline Barnebydendron riedelii (Tul.) JHKirkbr. & - & - & 0,97 & 65 & - & - & SI & Ane & LSL et al. 33 \\
\hline Bauhinia forficata Link & - & - & - & - & 2,82 & 28 & SI & Aut & LSL et al. 25 \\
\hline Copaifera lucens Dwyer & 5,39 & 15 & 1,10 & 49 & 2,81 & 29 & ST & Zoo & LSL et al. 31 \\
\hline Dalbergia nigra (Vell.) Allemão ex Benth. & 6,05 & 13 & 11,07 & 7 & 18,79 & 2 & SI & Ane & LSL et al. 30 \\
\hline Fabaceae sp.1 & - & - & - & - & 1,04 & 63 & $\mathrm{NC}$ & $\mathrm{NC}$ & LSL et al. 3 \\
\hline Fabaceae sp. 2 & - & - & 1,01 & 57 & - & - & $\mathrm{NC}$ & $\mathrm{NC}$ & LSL et al. 29 \\
\hline Fabaceae sp.3 & 0,81 & 84 & - & - & - & - & $\mathrm{NC}$ & $\mathrm{NC}$ & LSL et al. 4 \\
\hline Fabaceae sp. 4 & - & - & & & 0,93 & 72 & $\mathrm{NC}$ & NC & LSL et al. 117 \\
\hline Fabaceae sp. 5 & - & - & - & - & 0,92 & 75 & $\mathrm{NC}$ & $\mathrm{NC}$ & LSL et al. 9 \\
\hline Fabaceae sp.6 & 0,80 & 87 & - & - & - & - & $\mathrm{NC}$ & $\mathrm{NC}$ & LSL et al. 130 \\
\hline Fabaceae sp.7 & - & - & - & - & 1,43 & 51 & $\mathrm{NC}$ & $\mathrm{NC}$ & LSL et al. 92 \\
\hline Hymenaea courbaril L. & 1,64 & 48 & - & - & - & - & ST & Zoo & LSL et al. 8 \\
\hline Inga hispida Schott ex Benth. & 1,66 & 47 & - & - & - & - & $\mathrm{NC}$ & $\mathrm{NC}$ & LSL et al. 118 \\
\hline Lonchocarpus sericeus (Poir.) Kunth ex DC. & 5,03 & 18 & 2,65 & 24 & 0,89 & 84 & $\mathrm{NC}$ & NC & LSL et al. 36 \\
\hline Machaerium brasiliense Vogel & 1,13 & 59 & 3,42 & 18 & 4,62 & 18 & $\mathrm{NC}$ & $\mathrm{NC}$ & LSL et al. 116 \\
\hline Machaerium incorruptibile (Vell.) Benth. & 3,04 & 31 & - & - & 2,18 & 39 & SI & Ane & LSL et al. 135 \\
\hline Machaerium nyctitans (Vell.) Benth. & 0,82 & 78 & - & - & 3,03 & 25 & SI & Ane & LSL et al. 5 \\
\hline Machaerium paraguariense Hassl. & 7,42 & 10 & 1,89 & 36 & 1,85 & 44 & $\mathrm{Pi}$ & Ane & LSL et al. 143 \\
\hline Machaerium pedicellatum Vogel & - & - & 0,84 & 72 & 0,90 & 82 & NC & $\mathrm{NC}$ & LSL et al. 7 \\
\hline Mimosoideae sp.1 & 1,13 & 58 & - & - & - & - & NC & $\mathrm{NC}$ & LSL et al. 93 \\
\hline Myrocarpus frondosus Allemão & 2,51 & 35 & - & - & 11,84 & 5 & ST & Ane & LSL et al. 35 \\
\hline Parapiptadenia pterosperma (Benth.) Brenan & 4,78 & 21 & 27,48 & 2 & 8,94 & 7 & SI & Ane & LSL et al. 6 \\
\hline Peltophorum dubium (Spreng.) Taub. & 0,82 & 79 & 12,90 & 6 & - & - & SI & Ane & LSL et al. 34 \\
\hline
\end{tabular}




\begin{tabular}{|c|c|c|c|c|c|c|c|c|c|}
\hline \multirow{3}{*}{$\begin{array}{l}\text { Família / Espécie } \\
\text { Platypodium elegans Vogel }\end{array}$} & \multicolumn{6}{|c|}{ Sítios } & \multirow{3}{*}{$\begin{array}{l}\text { CSu } \\
\text { ST }\end{array}$} & \multirow{3}{*}{$\begin{array}{l}\text { SD } \\
\text { Ane }\end{array}$} & \multirow{3}{*}{$\begin{array}{c}\text { CN } \\
\text { LSL et al. } 122\end{array}$} \\
\hline & \multicolumn{2}{|c|}{ CS } & \multicolumn{2}{|c|}{$\mathbf{C C}$} & \multicolumn{2}{|c|}{ PA } & & & \\
\hline & - & - & - & - & 1,05 & 62 & & & \\
\hline Platymiscium floribundum Vogel & 0,88 & 70 & - & - & 3,43 & 22 & ST & Ane & LSL et al. 134 \\
\hline Platymiscium sp. & - & - & 1,23 & 44 & 1,90 & 42 & $\mathrm{NC}$ & $\mathrm{NC}$ & LSL et al. 144 \\
\hline Poeppigia procera C.Presl & - & - & - & - & 1,12 & 59 & SI & Ane & LSL et al. 129 \\
\hline $\begin{array}{l}\text { Pseudopiptadenia contorta (DC.) G.P.Lewis \& } \\
\text { M.P.Lima }\end{array}$ & 20 & 1 & 22,50 & 4 & 17,89 & 3 & SI & Ane & LSL et al. 140 \\
\hline Pseudopiptadenia sp. & - & - & 2,20 & 31 & 3,87 & 20 & $\mathrm{NC}$ & $\mathrm{NC}$ & LSL et al. 145 \\
\hline $\begin{array}{l}\text { Pseudopiptadenia warmingii (Benth.) G.P.Lewis } \\
\& \text { M.P.Lima }\end{array}$ & - & - & 1,11 & 48 & - & - & $\mathrm{Pi}$ & Ane & LSL et al. 114 \\
\hline $\begin{array}{l}\text { Senegalia kallunkiae (J.W.Grimes \& Barneby) } \\
\text { Seigler \& Ebinger }\end{array}$ & - & - & - & - & 0,89 & 85 & SI & Ane & LSL et al. 123 \\
\hline Senegalia sp. & 5,16 & 17 & 4,92 & 14 & 10,39 & 6 & $\mathrm{NC}$ & $\mathrm{NC}$ & LSL et al. 90 \\
\hline Swartzia acutifolia Vogel & 0,86 & 71 & 1,01 & 56 & - & - & ST & Zoo & LSL et al. 115 \\
\hline Zollernia glabra (Spreng.) Yakovlev & - & - & - & - & 1,60 & 49 & $\mathrm{NC}$ & $\mathrm{NC}$ & LSL et al. 91 \\
\hline \multicolumn{10}{|l|}{ LAURACEAE } \\
\hline Lauraceae sp. 1 & 5,17 & 16 & 0,98 & 63 & 1,87 & 43 & $\mathrm{NC}$ & Zoo & LSL et al. 128 \\
\hline Lauraceae sp. 2 & - & - & 1,33 & 40 & - & - & $\mathrm{NC}$ & $\mathrm{NC}$ & LSL et al. 142 \\
\hline Ocotea indecora (Schott) Mez & 1,02 & 63 & 0,96 & 70 & 1,4 & 54 & ST & Zoo & LSL et al. 89 \\
\hline \multicolumn{10}{|l|}{ LECYTHIDACEAE } \\
\hline Cariniana ianeirensis R.Knuth & 4,29 & 23 & - & - & 4,62 & 16 & ST & Ane & LSL et al. 47 \\
\hline Couratari asterotricha Prance & 0,80 & 88 & - & - & - & - & SI & Ane & LSL et al. 133 \\
\hline Couratari sp. & 0,81 & 83 & 1,13 & 47 & 0,92 & 77 & $\mathrm{NC}$ & $\mathrm{NC}$ & LSL et al. 113 \\
\hline Lecythidaceae sp. & - & - & 1,84 & 37 & - & - & $\mathrm{NC}$ & $\mathrm{NC}$ & LSL et al. 49 \\
\hline \multicolumn{10}{|l|}{ MALPIGHIACEAE } \\
\hline Bunchosia macilenta Dobson & 4,05 & 24 & - & - & 1,77 & 48 & ST & $\mathrm{NC}$ & LSL et al. 46 \\
\hline Malpighiaceae sp. & 1,07 & 61 & - & - & - & - & $\mathrm{NC}$ & $\mathrm{NC}$ & LSL et al. 126 \\
\hline \multicolumn{10}{|l|}{ MALVACEAE } \\
\hline Basiloxylon brasiliensis (All.) K.Schum. & - & - & 2,66 & 23 & 1,41 & 53 & ST & Ane & LSL et al. 112 \\
\hline Guazuma crinita Mart. cf. & 1,57 & 51 & - & - & - & - & $\mathrm{Pi}$ & Ane & LSL et al. 45 \\
\hline Eriotheca candolleana (K.Schum.) A.Robyns & 0,90 & 66 & - & - & - & - & SI & Ane & LSL et al. 94 \\
\hline Pseudobombax grandiflorum (Cav.) A.Robyns & 0,82 & 80 & 1,31 & 41 & - & - & $\mathrm{Pi}$ & Ane & LSL et al. 44 \\
\hline Pterygota brasiliensis Allemão & 4,83 & 20 & - & - & 4,89 & 15 & $\mathrm{NC}$ & $\mathrm{NC}$ & LSL et al. 48 \\
\hline \multicolumn{10}{|l|}{ MELIACEAE } \\
\hline Cabralea sp. & - & - & 1,01 & 58 & - & - & $\mathrm{NC}$ & Zoo & LSL et al. 87 \\
\hline Cedrela fissilis Vell. & 2,54 & 34 & - & - & - & - & SI & Zoo & LSL et al. 109 \\
\hline Guarea guidonia (L.) Sleumer & 0,82 & 76 & 2,29 & 29 & 0,98 & 67 & SI & Zoo & LSL et al. 40 \\
\hline Meliaceae sp. & 0,81 & 82 & - & - & - & - & $\mathrm{NC}$ & Zoo & LSL et al. 95 \\
\hline Trichilia casaretti C.DC. & 4,46 & 22 & 9,39 & 9 & 8,18 & 8 & ST & Zoo & LSL et al. 86 \\
\hline Trichilia clausseni C.DC. & - & - & 2,08 & 34 & - & - & ST & Zoo & LSL et al. 41 \\
\hline Trichilia hirta L. & 1,33 & 55 & - & - & 1,53 & 50 & ST & Zoo & LSL et al. 42 \\
\hline
\end{tabular}




\begin{tabular}{|c|c|c|c|c|c|c|c|c|c|}
\hline \multirow{3}{*}{$\begin{array}{l}\text { Família / Espécie } \\
\text { Trichilia lepidota Mart. }\end{array}$} & \multicolumn{6}{|c|}{ Sítios } & \multirow{3}{*}{$\begin{array}{l}\text { CSu } \\
\text { ST }\end{array}$} & \multirow{3}{*}{$\begin{array}{l}\text { SD } \\
\text { Zoo }\end{array}$} & \multirow{3}{*}{$\begin{array}{c}\text { CN } \\
\text { LSL et al. } 108\end{array}$} \\
\hline & \multicolumn{2}{|c|}{ CS } & \multicolumn{2}{|c|}{$\mathbf{C C}$} & \multicolumn{2}{|c|}{ PA } & & & \\
\hline & 1,96 & 40 & - & - & 0,92 & 73 & & & \\
\hline Trichilia pallida $\mathrm{Sw}$. & 1,53 & 52 & 1,21 & 46 & 2,24 & 38 & SI & Zoo & LSL et al. 36 \\
\hline Trichilia pseudostipularis (A.Juss.) C.DC. & 3,01 & 32 & 0,99 & 60 & 16,30 & 4 & ST & Zoo & LSL et al. 43 \\
\hline Trichilia silvatica C.DC. & - & - & 5,59 & 13 & 0,88 & 93 & ST & Zoo & LSL et al. 88 \\
\hline Trichilia sp. & 1,63 & 49 & - & - & 3,77 & 21 & $\mathrm{NC}$ & Zoo & LSL et al. 85 \\
\hline \multicolumn{10}{|l|}{ MONIMIACEAE } \\
\hline Siparuna guianensis Aubl. & - & - & 2,45 & 25 & - & - & SI & Zoo & LSL et al. 50 \\
\hline \multicolumn{10}{|l|}{ MORACEAE } \\
\hline Brosimum glaziovii Taub. & - & - & 2,36 & 26 & 1,94 & 41 & SI & Zoo & LSL et al. 37 \\
\hline Brosimum guianense (Aubl.) Huber & 1,74 & 44 & 2,93 & 21 & 0,95 & 69 & SI & Zoo & LSL et al. 75 \\
\hline Ficus gomelleira Kunth & 2,44 & 37 & - & - & - & - & ST & Zoo & LSL et al. 51 \\
\hline Sorocea guilleminiana Gaudich. & 1,09 & 60 & - & - & - & - & SI & Zoo & LSL et al. 98 \\
\hline \multicolumn{10}{|l|}{ MYRTACEAE } \\
\hline Eugenia sp. 1 & - & - & 3,05 & 20 & 0,89 & 88 & $\mathrm{NC}$ & Zoo & LSL et al. 73 \\
\hline Eugenia sp.2 & - & - & 1,25 & 42 & - & - & $\mathrm{NC}$ & Zoo & LSL et al. 38 \\
\hline Eugenia sp.3 & 0,83 & 74 & - & - & - & - & $\mathrm{NC}$ & Zoo & LSL et al. 70 \\
\hline Myrcia eumecephylla (O.Berg) Nied. & 0,80 & 86 & - & - & 3,34 & 23 & $\mathrm{NC}$ & Zoo & LSL et al. 104 \\
\hline \multicolumn{10}{|l|}{ NYCTAGINACEAE } \\
\hline Guapira noxia (Netto) Lundell & - & - & - & - & 2,60 & 32 & SI & Zoo & LSL et al. 84 \\
\hline Guapira opposita (Vell.) Reitz & 0,90 & 67 & 6,02 & 11 & 0,91 & 80 & SI & Zoo & LSL et al. 39 \\
\hline \multicolumn{10}{|l|}{ OCHNACEAE } \\
\hline Ouratea sp. & 0,82 & 75 & - & - & - & - & $\mathrm{NC}$ & $\mathrm{NC}$ & LSL et al. 71 \\
\hline \multicolumn{10}{|l|}{ POLYGONACEAE } \\
\hline Coccoloba alnifolia Casar. & - & - & 0,99 & 61 & - & - & SI & Zoo & LSL et al. 52 \\
\hline Coccoloba striata Benth. & - & - & - & - & 0,90 & 81 & $\mathrm{NC}$ & Zoo & LSL et al. 107 \\
\hline Ruprechtia laxiflora Meisn. & - & - & - & - & 2,29 & 36 & SI & Ane & LSL et al. 74 \\
\hline \multicolumn{10}{|l|}{ PROTEACEAE } \\
\hline Euplassa sp. & - & - & - & - & 0,88 & 91 & $\mathrm{NC}$ & $\mathrm{NC}$ & LSL et al. 72 \\
\hline \multicolumn{10}{|l|}{ RHAMNACEAE } \\
\hline Ziziphus glaziovii Warm. & - & - & - & - & 1,01 & 66 & ST & Zoo & LSL et al. 103 \\
\hline Ziziphus joazeiro Mart. & 3,11 & 30 & - & - & - & - & SI & Zoo & LSL et al. 53 \\
\hline \multicolumn{10}{|l|}{ RUBIACEAE } \\
\hline Alseis pickelii Pilg. \& Schmale & 8,78 & 9 & 23,91 & 3 & 23,81 & 1 & ST & Zoo & LSL et al. 57 \\
\hline Bathysa sp. & - & - & 2,21 & 30 & 1,25 & 56 & $\mathrm{NC}$ & $\mathrm{NC}$ & LSL et al. 54 \\
\hline Rubiaceae sp. & - & - & - & - & 2,33 & 34 & $\mathrm{NC}$ & $\mathrm{NC}$ & LSL et al. 68 \\
\hline \multicolumn{10}{|l|}{ RUTACEAE } \\
\hline Almeidea rubra A.St.-Hil. & 1,85 & 42 & - & - & 2,31 & 35 & ST & Aut & LSL et al. 83 \\
\hline Balfourodendron riedelianum (Engl.) Engl. & - & - & 2,19 & 32 & - & - & SI & Ane & LSL et al. 55 \\
\hline $\begin{array}{l}\text { Neoraputia alba (Nees \& Mart.) Emmerich ex } \\
\text { Kallunki }\end{array}$ & 11,00 & 8 & - & - & 2,66 & 31 & ST & Aut & LSL et al. 67 \\
\hline
\end{tabular}




\begin{tabular}{|c|c|c|c|c|c|c|c|c|c|}
\hline \multirow{3}{*}{$\begin{array}{l}\text { Família / Espécie } \\
\text { Zanthoxylum rhoifolium Lam. }\end{array}$} & \multicolumn{6}{|c|}{ Sítios } & \multirow{3}{*}{$\begin{array}{c}\text { CSu } \\
\mathrm{Pi}\end{array}$} & \multirow{3}{*}{$\begin{array}{l}\text { SD } \\
\text { Zoo }\end{array}$} & \multirow{3}{*}{$\begin{array}{c}\text { CN } \\
\text { LSL et al. } 81\end{array}$} \\
\hline & \multicolumn{2}{|c|}{ CS } & \multicolumn{2}{|c|}{$\mathbf{C C}$} & \multicolumn{2}{|c|}{ PA } & & & \\
\hline & 3,52 & 27 & - & - & - & - & & & \\
\hline \multicolumn{10}{|l|}{ SALICACEAE } \\
\hline Casearia sp.1 & 0,89 & 68 & 4,75 & 15 & 0,92 & 74 & $\mathrm{NC}$ & $\mathrm{NC}$ & LSL et al. 69 \\
\hline Casearia sp. 2 & 1,27 & 56 & 1,98 & 35 & 5,65 & 14 & $\mathrm{NC}$ & $\mathrm{NC}$ & LSL et al. 99 \\
\hline Casearia sylvestris $\mathrm{Sw}$. & - & - & 1,02 & 55 & 2,49 & 33 & SI & Zoo & LSL et al. 82 \\
\hline Macrothumia kuhlmannii (Sleumer) M.H.Alford & 1,40 & 53 & - & - & - & - & ST & Ane & LSL et al. 56 \\
\hline \multicolumn{10}{|l|}{ SAPINDACEAE } \\
\hline $\begin{array}{l}\text { Allophylus edulis (A.St.-Hil. et al.) Hieron. ex } \\
\text { Niederl. }\end{array}$ & 2,99 & 33 & - & - & 1,02 & 64 & $\mathrm{Pi}$ & Zoo & LSL et al. 100 \\
\hline Cupania sp. & - & - & - & - & 2,86 & 27 & $\mathrm{NC}$ & $\mathrm{NC}$ & LSL et al. 105 \\
\hline Matayba sp. & 1,61 & 50 & 1,03 & 54 & - & - & $\mathrm{NC}$ & $\mathrm{NC}$ & LSL et al. 58 \\
\hline Melicoccus oliviformis Kunth & - & - & - & - & 0,98 & 68 & SI & $\mathrm{NC}$ & LSL et al. 76 \\
\hline \multicolumn{10}{|l|}{ SAPOTACEAE } \\
\hline $\begin{array}{l}\text { Chrysophyllum gonocarpum (Mart. \& Eichler ex } \\
\text { Miq.) Engl. }\end{array}$ & 0,83 & 73 & 0,97 & 66 & 1,82 & 45 & ST & Zoo & LSL et al. 126 \\
\hline Chrysophyllum lucentifolium Cronquist & - & - & 1,22 & 45 & 0,89 & 86 & ST & Zoo & LSL et al. 102 \\
\hline Manilkara salzmannii (A.DC.) H.J.Lam & 1,34 & 54 & - & - & - & - & ST & Zoo & LSL et al. 125 \\
\hline Pouteria guianensis Aubl. & - & - & 0,96 & 69 & - & - & ST & Zoo & LSL et al. 131 \\
\hline Pouteria sp. & - & - & 1,24 & 43 & 0,94 & 70 & $\mathrm{NC}$ & Zoo & LSL et al. 106 \\
\hline Sapotaceae sp.1 & - & - & - & - & - & - & $\mathrm{NC}$ & Zoo & LSL et al. 59 \\
\hline Sapotaceae sp.2 & 1,98 & 39 & - & - & - & - & $\mathrm{NC}$ & Zoo & LSL et al. 64 \\
\hline Sapotaceae sp.3 & 1,91 & 41 & - & - & 0,89 & 87 & $\mathrm{NC}$ & Zoo & LSL et al. 77 \\
\hline \multicolumn{10}{|l|}{ ULMACEAE } \\
\hline Ampelocera glabra Kuhlm. & 2,47 & 36 & - & - & - & - & ST & Zoo & LSL et al. 60 \\
\hline VERBENACEAE & & & & & & & & & LSL et al. 78 \\
\hline Verbenaceae sp. & - & - & 0,96 & 71 & - & - & $\mathrm{NC}$ & $\mathrm{NC}$ & LSL et al. 65 \\
\hline \multicolumn{10}{|l|}{ INDETERMINADA } \\
\hline Indeterminada 1 & 0,80 & 89 & - & - & - & - & $\mathrm{NC}$ & $\mathrm{NC}$ & LSL et al. 96 \\
\hline Indeterminada 2 & - & - & - & - & 1,0 & 65 & $\mathrm{NC}$ & $\mathrm{NC}$ & LSL et al. 61 \\
\hline Indeterminada 3 & - & - & 1,1 & 50 & - & - & $\mathrm{NC}$ & $\mathrm{NC}$ & LSL et al. 66 \\
\hline Indeterminada 4 & - & - & 0,97 & 67 & - & - & $\mathrm{NC}$ & $\mathrm{NC}$ & LSL et al. 101 \\
\hline Indeterminada 5 & 0,80 & 87 & - & - & 1,13 & 58 & $\mathrm{NC}$ & $\mathrm{NC}$ & LSL et al. 111 \\
\hline Indeterminada 6 & - & - & - & - & 0,92 & 79 & $\mathrm{NC}$ & $\mathrm{NC}$ & LSL et al. 62 \\
\hline Indeterminada 7 & - & - & - & - & 0,88 & 92 & $\mathrm{NC}$ & $\mathrm{NC}$ & LSL et al. 79 \\
\hline Indeterminada 8 & - & - & - & - & 0,93 & 71 & $\mathrm{NC}$ & $\mathrm{NC}$ & LSL et al. 141 \\
\hline Indeterminada 9 & - & - & - & - & 1,78 & 46 & $\mathrm{NC}$ & $\mathrm{NC}$ & LSL et al. 110 \\
\hline Indeterminada 10 & 0,84 & 72 & - & - & - & - & $\mathrm{NC}$ & $\mathrm{NC}$ & LSL et al. 132 \\
\hline Indeterminada 11 & 0,80 & 90 & - & - & - & - & $\mathrm{NC}$ & $\mathrm{NC}$ & LSL et al. 63 \\
\hline Indeterminada 12 & - & - & 1 & 59 & - & - & $\mathrm{NC}$ & $\mathrm{NC}$ & LSL et al. 80 \\
\hline Indeterminada 13 & 0,89 & 69 & - & - & - & - & $\mathrm{NC}$ & $\mathrm{NC}$ & LSL et al. 97 \\
\hline
\end{tabular}


quais podem ser consideradas como "localmente raras" (Abreu et al. 2013) representando 36\% da riqueza e $4 \%$ do total de indivíduos, merecendo maior atenção quanto à sua conservação (Silva et al. 2003). A diversidade de espécies nos sítios foi de 3,73 no CS, 3,58 no CC e 4,21 no PA. Apesar dos três sítios terem sido perturbados no passado, os valores de diversidade apresentados são considerados altos e semelhantes aos encontrados em FES em estágio sucessional mais avançado (Lopes et al. 2002; Souza et al. 2003; Dalanesi et al. 2004; Carvalho et al. 2007; Gusson et al. 2009; Archanjo et al. 2012).

A curva de rarefação para a riqueza de espécies formou dois grupos demonstrando que os sítios CS e PA possuem maior riqueza em relação ao sítio CC (Fig. 2a). O mesmo resultado foi encontrado para a diversidade de espécies (Fig. 2b). Os maiores valores de diversidade encontrados nos sítios CS e PA podem ser explicados pelo destaque de poucas espécies nessas áreas. Ao contrário
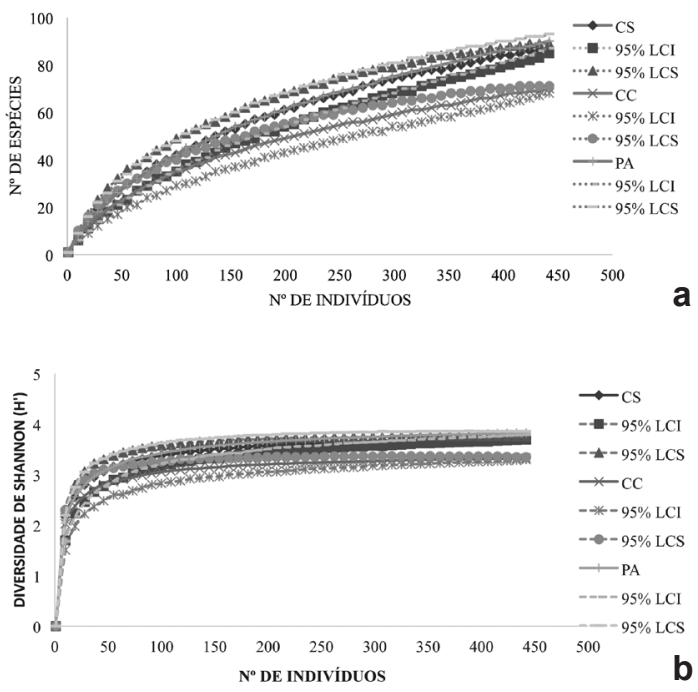

Figura 2 - a-b. Curvas de rarefação de indivíduos baseadas em intervalos de $95 \%$ de confiança, no fragmento florestal, município de Alegre-ES, dos sítios e parcelas - a. para a riqueza de espécies; b. para índice diversidade de Shannon. (CS = sítio de corte seletivo; $\mathrm{CC}=$ sítio de cultivo de café; $\mathrm{PA}=$ sítio de pastagens; LCS = limite de confiança Superior; LCI = limite de confiança inferior).

Figure 2 - $a-b$. Rarefaction curves of individuals based on $95 \%$ confidence intervals, in the forest fragment, Alegre-ES, of the sites and plots $-a$. for species richness; $b$. Shannon diversity index. (CS $=$ selective cutting site $\mathrm{CC}=$ coffee cultivation site $\mathrm{PA}=$ pasture site; $\mathrm{LCS}=$ upper confidence limit; $\mathrm{LCI}=$ lower confidence limit). disso, acreditamos que houve uma concentração relativamente elevada de abundância de uma espécie, sendo essa a Apuleia leiocarpa (Vogel) JFMacbr, no sítio CC, o que pode ter influenciado no baixo valor de diversidade nessa área.

Foram registradas quatorze espécies ameaçadas de extinção pelo IUCN (1), Livro Vermelho da Flora $\left({ }^{2}\right)$ e IEMA $\left({ }^{3}\right)$, a saber: Amburana cearenses (Allemão) A.C.Sm. ${ }^{1,2}$, Apuleia leiocarpa (Vogel) JFMacbr. ${ }^{2}$, Brosimum glaziovii Taub. ${ }^{1}$, Bunchosia macilenta Dobson ${ }^{2}$, Cariniana ianeirensis R.Knuth ${ }^{1,2}$, Cedrela fissilis Vell. ${ }^{1,2}$, Couratari asterotricha Prance ${ }^{1,2,3}$, Dalbergia nigra (Vell.) Allemão ex Benth. ${ }^{1}$, ${ }^{2}$, Inga hispida Schott ex Benth. ${ }^{1}$, Paratecoma peroba (Record) Kuhlm. ${ }^{2}$, Sorocea guilleminiana Gaudich ${ }^{1}$, Terminalia mameluco Picke ${ }^{3}$, Trichilia silvatica C.DC. ${ }^{1}$ e Trichilia casaretti C.DC. ${ }^{1}$. Esse fato ressalta a relevância do fragmento florestal estudado e indica a necessidade de sua conservação para a manutenção da biodiversidade regional (Carvalho et al. 2007; Dan et al. 2010; Archanjo et al. 2012).

A espécie ameaçada $A$. cearenses, apesar de ser típica da região semi-árida nordestina (Guedes et al. 2012), tem registro de ocorrência em florestas estacionais nos estados do Rio de Janeiro (Ribeiro \& Lima 2009) e Minas Gerais (Arruda et al. 2013), além do presente estudo. Isso reforça a indicação de que $A$. cearensis ocorre não somente na região nordestina do Brasil, mas também nas regiões central e sudeste com uma distribuição mais associada às florestas estacionais (Ribeiro \& Lima 2009). Assim, acredita-se que $A$. cearensis seguiu uma rota migratória por meio de um provável corredor formado durante a última glaciação, tendo a Caatinga como um nódulo migratório.

Vale ressaltar a presença de C. ianeirensis, espécie para a qual não havia registro de ocorrência na flora do estado do Espírito Santo até 2013 (Martinelli \& Moraes 2013). Essa informação demonstra o quanto é necessário ampliar os estudos florísticos no Espírito Santo. C. ianeirensis possui alto valor econômico, sendo explorada para uso de sua madeira (Braga et al. 2011).

Com relação aos valores de importância (VI) (Tab. 1) em cada sítio, Pseudopiptadenia contorta (DC.) G.P.Lewis \& M.P.Lima destacou-se no sítio $\mathrm{CS}$, ficando em primeiro lugar na colocação. Essa espécie é categorizada como secundária inicial e descrita como ruderal em alguns trabalhos (Dan et al. 2010). Assim, o seu destaque pode estar relacionado ao histórico desse sítio, pois o corte 
seletivo de espécies arbóreas pode favorecer o desenvolvimento de espécies de início de sucessão no povoamento (Carvalho \& Nascimento 2009). Foi observada uma grande representatividade dessa espécie em outros trabalhos realizados no sul do estado do Espírito Santo (Archanjo et al. 2012; Abreu et al. 2013; Silva et al. 2017), por isso, os resultados sugerem que $P$. contorta é uma espécie característica das matas dessa região.

Verificamos uma alta representatividade de espécies pertencentes à família Euphorbiaceae dentre as dez primeiras espécies na colocação no sítio CS, podendo algumas espécies dessa família serem indicadoras de ambientes perturbados (Moreira et al. 2007), a saber: Actinostemon verticillatus (Klotzsch) Baill., Pachystroma longifolium (Nees) I.M.Johnst., Actinostemon concolor (Spreng.) Müll.Arg. e Cnidoscolus oligandrus var. xerophilus A. de Mattos Filho \& Rizzini, as quais representam $26,6 \%$ da densidade desse sítio.

Dentre as dez primeiras espécies na colocação do sítio CS, há presença de espécies secundárias tardias, a saber: Astronium graveolens Jacq., Neoraputia alba (Nees \& Mart.) Emmerich ex Kallunki e Alseis pickelii Pilg. \& Schmale, o que pode indicar que o respectivo sítio está se recuperando da perturbação causada pelo corte seletivo e está avançando em seu estágio sucessional (Franco et al. 2014).

No sítio CC houve um destaque em VI da espécie secundária inicial $A$. leiocarpa como primeira colocada (Tab. 1), fato que se deve à alta abundância dessa espécie nessa área. A espécie que ficou em segundo lugar na colocação é Parapiptadenia pterosperma (Benth.) Brenan, essa espécie também é categorizada com secundária inicial, assim pode estar favorecendo o ambiente para o desenvolvimento de espécies de final de sucessão. A maior importância de A. leiocarpa e $P$. pterosperma nesse sítio está relacionada à localização das parcelas próximas à borda, local propício para o desenvolvimento de espécies de início de sucessão (Tabarelli et al. 2010). Espécies de início de sucessão e da família Fabaceae também foram as mais importantes em uma floresta regenerada pós abandono de monocultura do café (López-Gómez et al. 2008; Oliveira-Neto et al. 2017). Esses autores acreditam que agricultores podem ter permitido a coexistência dessas espécies com o cultivo de café com o intuito de sombreamento e fixação de nitrogênio no solo. Assim, após o abandono e tendo essas espécies como matrizes, suas sementes disseminaram-se localmente.

Em terceiro lugar na colocação no sítio CC, A. pickelii destacou-se com alta densidade e frequência. Essa é uma espécie secundária tardia que auxilia na composição do dossel, não atingindo grande porte e sendo indicadora de ambientes com certo grau de conservação (Rabelo et al. 2012). É relevante ressaltar a alta densidade de Erythroxylum pulchrum A.St.-Hil. e T. casaretti, que juntas, perfazem $14,5 \%$ da densidade total nesse sítio. Essas duas espécies além de serem secundárias tardias, são zoocóricas, corroborando com LópezGómez et al. (2008), que também encontraram grande abundância de indivíduos zoocóricos em florestas provenientes de abandono de cultivo de café. A presença dessas espécies é um indicativo de que há alimento disponível para a fauna local, contribuindo para a manutenção da biodiversidade local.

No sítio PA, A. pickelii obteve maior VI e primeiro lugar na colocação (Tab. 1), sendo essa uma espécie secundária tardia e sensível a perturbações (Rabelo et al. 2012). Apesar desse sítio ter regenerando-se de pastagem e possuir menor tempo de pousio em relação aos demais sítios, o destaque dessa espécie pode ter relação com a maior proteção aos efeitos de borda e aos efeitos antrópicos observados no sítio PA (Tabarelli et al. 2010). O sítio PA tem maior proteção em relação aos sítios CS e CC, com a maior parte da sua extensão circundada pelos demais sítios e dentro dos limites do PEAMA, onde conta com certa fiscalização. Em segundo lugar está a espécie $D$. nigra, considerada de baixa especificidade e de alta amplitude de tolerância ambiental (Carvalho et al. 2006). Já em terceiro lugar encontra-se $P$. contorta, a qual ficou bem posicionada devido aos seus indivíduos remanescentes possuírem grande porte, o que refletiu nos valores de dominância e na sua colocação no sítio PA, onde foi encontrada baixa densidade dessa espécie. A mesma situação foi verificada por Lopes et al. (2002) e, segundo esses autores, a baixa densidade de uma determinada espécie pode indicar que a mesma apresenta-se com dificuldades de adaptação ao atual estágio sucessional de uma área, sendo menos competitiva com relação às condições momentâneas do ambiente. Contudo, acreditamos que logo após o abandono da pastagem para regeneração, $P$. contorta contribuiu modificando o ambiente para o recrutamento de espécies secundárias tardias e zoocóricas. 
Em quarto lugar na colocação está Trichilia pseudostipularis (A.Juss.) C.DC., fortemente recrutada nesse sítio, apresentando maior valor de densidade (10\%) em relação a todas as espécies do sítio PA. A espécie T. casaretti ficou em oitavo lugar na colocação. A presença de T. pseudostipularis e $T$. casaretti é relevante para o sítio PA, pois as mesmas são secundarias tardias e zoocóricas.

Verificamos que os três sítios possuem poucas espécies em comuns dentre as dez mais importantes, sendo essas: Astronium concinnum Schott, A. pickelii e P. contorta e P. contorta no sítio CS e A. leiocarpa (Tab. 1). As diferenças entre as espécies mais importantes nos três sítios podem estar relacionadas à heterogeneidade ambiental, a qual pode ocorrer em um mesmo fragmento florestal e nesse caso, pode estar relacionada à influência do uso anterior do solo e ao estágio sucessional de cada sítio que refletem fatores como: pressão antrópica, face da encosta, efeito de borda, solo (Braga et al. 2015), entre outros. Além disso, Lugo \& Helmer et al. (2004) salientam que florestas regeneradas de cultivo agrícola podem apresentar composição florística diferente das florestas do entorno.

Do total, $55 \%$ das espécies foram amostradas em apenas um dos sítios e $20 \%$ das espécies foram comuns aos três sítios. Analisando a similaridade qualitativa de espécies por meio do índice de Sørensen, os valores entre os sítios variaram entre 0,47 e 0,55 (Fig. 3a). O primeiro bloco foi formado entre os sítios CS e PA, demonstrando maior similaridade florística. A vegetação do sítio CS pode ter influenciado, em maior proporção, a vegetação do sítio PA em decorrência da proximidade espacial entre esses sítios. A dissimilaridade florística entre os sítios CS e PA em relação ao sítio CC pode estar relacionada aos respectivos históricos de perturbação e aos fatores ambientais que podem ter influenciado no decorrer do processo de regeneração de cada área.

Dentre os fatores ambientais, verificamos que o sítio CC possui sua face de exposição solar voltada à vertente sul e os sítios CS e PA estão voltadas às vertentes norte e leste, respectivamente. Áreas com vertentes voltadas à vertente sul tendem a receber menor radiação solar, a qual influencia na acidez e nutrientes do solo, que por sua vez podem influenciar na vegetação (Braga et al. 2015). Contudo, mesmo que o sítio CS e PA fossem floristicamente semelhantes, a estrutura pode se comportar de forma independente (Durigan et al. 2008), conforme encontrado na análise de similaridade de Morisita Horn, que considera a abundância de indivíduos por espécie. Nessa análise, houve formação de diferentes blocos florísticos no dendrograma, demonstrando que os sítios CC e PA $(0,46)$ possuem maior similaridade (Fig. 3b). Acreditamos que essa similaridade estrutural está relacionada, principalmente, ao estágio sucessional (Lopes et al. 2002), pois o sítio CS foi o único que não sofreu corte raso, podendo inferir que seu estágio sucessional difere dos demais sítios (Morel et al. 2016). Assim, acreditamos que algumas espécies ocorrentes no sítio CS estão iniciando sua colonização no sítio PA, e os indivíduos de maior abundância no sítio PA são comuns ao sítio CC.

Em relação à categoria sucessional (Tab. 2), a maior densidade de secundárias tardias nos sítios CS e PA influenciou nos valores de VC e VI dos mesmos visto que, na dominância, as espécies tardias não se sobressaíram, indicando que há abundância de indivíduos de menor porte nessa categoria sucessional. As condições ambientais nos sítios CS e PA podem estar favoráveis ao desenvolvimento das secundárias tardias mais jovens, as quais não atingiram seu potencial em dominância, apontando que essas áreas estão caminhando para um estágio avançado de sucessão e com potencial para avançar mais (Franco et al. 2014).
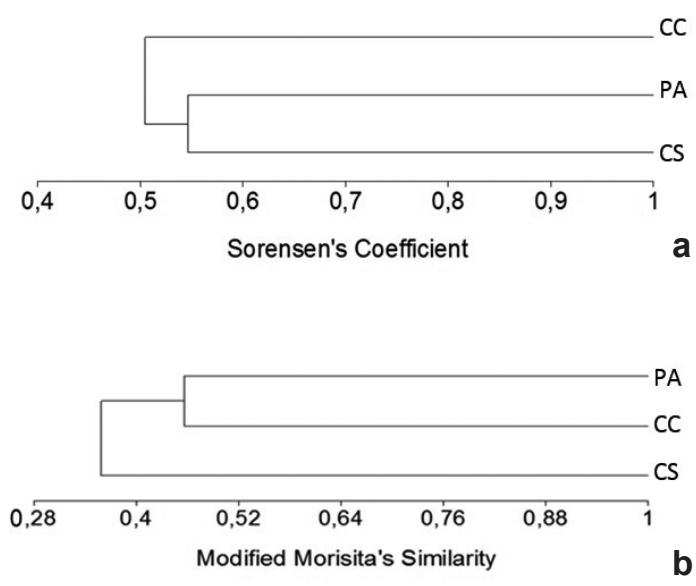

Figura 3-a-b. Dendrogramas de similaridade de espécies arbóreas entre os sítios amostrados no fragmento florestal, município de Alegre-ES, dos sítios e parcelas, por meio do método de agrupamento pela média de grupo (UPGMA) - a. a partir do índice de Sørensen; b. a partir do índice de Morisita Horn. ( $\mathrm{CS}=$ sítio de corte seletivo; $\mathrm{CC}=$ sítio de cultivo de café; $\mathrm{PA}=$ sítio de pastagens).

Figure 3-a-b. Dendrograms of similarity of arboreal species between the sites sampled in the forest fragment, Alegre-ES municipality, by means of the group mean grouping method (UPGMA) - a. using the Sørensen indice; $b$. using the Morisita Horn indice. (CS = selective cutting site; $\mathrm{CC}=$ coffee growing site; $\mathrm{PA}=$ pasture site). 
Tabela 2 - Parâmetros fitossociológicos por categoria sucessional de espécies arbóreos com DAP $\geq 5 \mathrm{~cm}$ amostradas em três sítios que compõem o Fragmento florestal, município de Alegre-ES $-\mathrm{CSu}=$ Categoria sucessional; $\mathrm{Pi}=$ pioneira; $\mathrm{SI}=$ secundáia inicial; $\mathrm{ST}=$ secundaria tardia $; \mathrm{CS}=$ sítio de corte seletivo $\mathrm{CC}=$ sítio de cultivo de café; $\mathrm{PA}=$ sítio de pastagens; $\mathrm{DR}$ $=$ densidade relativa; $\mathrm{DoR}=$ dominância relativa; $\mathrm{FR}=$ frequência relativa; $\mathrm{VC}=$ valor de cobertura; $\mathrm{VI}=$ valor de importância.

Table 2 - Phytosociological parameters by successional category of arboreal species with $\mathrm{DBH} \geq 5 \mathrm{~cm}$ sampled at three sites that make up the forest fragment, Alegre-ES $-\mathrm{CSu}=$ Succession category; $\mathrm{Pi}=$ pioneer; $\mathrm{SI}=$ initial secundary; $\mathrm{ST}=$ secondary school; $\mathrm{CS}=$ selective cutting site; $\mathrm{CC}=$ coffee growing site; $\mathrm{PA}=$ pasture site; $\mathrm{DR}=$ relative density; $\mathrm{DoR}=$ relative dominance; $\mathrm{FR}=$ relative frequency; $\mathrm{VC}=$ coverage value; $\mathrm{VI}=$ importance value.

\begin{tabular}{|c|c|c|c|c|c|c|c|c|c|c|c|c|c|c|c|}
\hline \multirow{2}{*}{$\mathrm{CSu}$} & \multicolumn{3}{|c|}{ DR (\%) } & \multicolumn{3}{|c|}{ DoR (\%) } & \multicolumn{3}{|c|}{ FR (\%) } & \multicolumn{3}{|c|}{ VC (\%) } & \multicolumn{3}{|c|}{ VI (\%) } \\
\hline & CS & $\mathrm{CC}$ & PA & CS & $\mathrm{CC}$ & PA & CS & CC & PA & CS & CC & PA & CS & $\mathrm{CC}$ & PA \\
\hline Pi & 9,3 & 1,3 & 2,1 & 15,6 & 1,2 & 3,9 & 25,0 & 10,0 & 14,3 & 12,5 & 1,2 & 3,0 & 16,6 & 4,2 & 6,8 \\
\hline SI & 27,5 & 47,3 & 27,9 & 36,3 & 65,4 & 40,8 & 25,0 & 30,0 & 28,6 & 31,9 & 56,3 & 34,4 & 29,6 & 47,5 & 32,4 \\
\hline ST & 50,9 & 36,6 & 46,2 & 32,0 & 24,0 & 36,3 & 25,0 & 30,0 & 28,6 & 41,4 & 30,3 & 41,3 & 36,0 & 30,2 & 37,0 \\
\hline
\end{tabular}

Os parâmetros fitossociológicos, estabelecidos com base nas categorias sucessionais, apresentaram todos os valores relativamente maiores para a secundárias iniciais no sítio $\mathrm{CC}$, demonstrando a representatividade desse grupo e sinalizando um ambiente com estágio relativamente menos avançado de sucessão. Assim, o sítio CC é considerado categorizado com estágio intermediário de regeneração e maior grau relativo de perturbação devido à sua área de borda. Pode-se inferir que as espécies secundárias iniciais estão proporcionando condições favoráveis para o recrutamento de espécies exigentes (Braga et al. 2011), contribuindo para o avanço sucessional dessa área.

A zoocoria e a anemocoria foram bem representadas no fragmento florestal todo, sendo que a zoocoria obteve os maiores valores nos três sítios quando considerada a riqueza de espécies $(\mathrm{CS}=41 \%, \mathrm{CC}=41 \%$ e $\mathrm{PA}=33 \%)($ Fig. $4 \mathrm{a})$ e considerando a abundância, destacou-se somente no sítio PA (38\%). A abundância de indivíduos zoocóricos indica maior atividade relativa da fauna dispersora em ambientes mais conservados (Carvalho 2010) e com menor perturbação (Gusson et al. 2009). No caso do sítio PA isso pode estar relacionado com a localização de sua área no interior do fragmento. A anemocoria obteve maior proporção entre os indivíduos no sítio CS (30\%) e CC (47\%) (Fig. 4b). A anemocoria aponta menor presença relativa de sub-bosque e menor sucessão ecológica (Carvalho 2010), situação encontrada em algumas parcelas dos sítios CS e CC que estão localizadas em bordas, as quais contribuíram para esses resultados.

Com relação à área basal, a mesma variou de $27,21 \mathrm{~m}^{2} /$ ha no sítio PA a $32,33 \mathrm{~m}^{2} /$ ha no sítio CC, sendo que o sítio CS apresentou valor intermediário, no caso $27,52 \mathrm{~m}^{2} / \mathrm{ha}$ (Tab. 3). Esses valores são atribuídos, pelo CONAMA (1994) para florestas em estágio avançado de regeneração no Espírito Santo
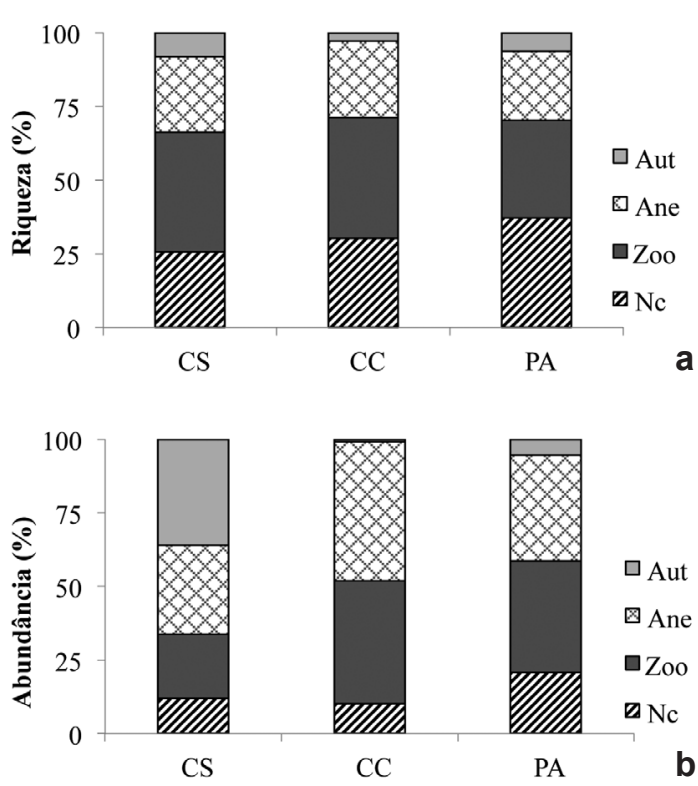

Figura 4 - a-b. Síndromes de dispersão no fragmento florestal, município de Alegre-ES - a. considerando a riqueza; b. considerando a abundância. $(\mathrm{CS}=$ sítio de corte seletivo; $\mathrm{CC}=$ sítio de cultivo de café; $\mathrm{PA}=$ sítio de pastagens; Ane = anemocoria; $\mathrm{Aut}=$ autocoria; $\mathrm{Zoo}=$ zoocoria; $\mathrm{NC}=$ não caracterizada por carência de informações pertinentes).

Figure $4-\mathrm{a}-\mathrm{b}$. Dispersion syndromes in the forest fragment, Alegre$\mathrm{ES}-\mathrm{a}$. considering richness; $\mathrm{b}$. considering abundance. $(\mathrm{CS}=$ selective cutting site; $\mathrm{CC}=$ coffee growing site; $\mathrm{PA}=$ pasture site; Ane = anemocoria; $\mathrm{Aut}=$ autocoria; $\mathrm{Zoo}=$ zoocoria; $\mathrm{NC}=$ not characterized due to lack of pertinent information. 
Tabela 3 - Os valores médios ( \pm desvio padrão) da área basal (AB) para indivíduos arbóreos com $\mathrm{DAP} \geq 5 \mathrm{~cm}$ amostradas em três sítios que compõem o Fragmento florestal, município de Alegre-ES. (CS = sítio corte seletivo; $\mathrm{CC}=$ sítio de cultivo de café; $\mathrm{PA}=$ sítio de pastagens. Onde as análises de variância indicaram diferenças significativas, médias seguidas por letras diferentes na coluna não diferiram significativamente entre si pelo teste de Tukey em $\mathrm{P} \leq 0,05$ ).

Table 3 - The mean values ( \pm standard deviation) of basal area (AB) for tree individuals with $\mathrm{DBH} \geq 5 \mathrm{~cm}$ sampled at three sites that make up the Forest Fragment, Alegre-ES municipality. ( $C S=$ selective court site; $\mathrm{CC}=$ coffee growing site; $\mathrm{PA}=$ pasture site. Where analyzes of variance indicated significant differences, mean values followed by different letters in the column did not differ significantly from each other by the Tukey test at $\mathrm{P} \leq 0.05$ ).

\begin{tabular}{cc}
\hline Sítio & $\mathbf{A B}\left(\mathbf{m}^{2} / \mathbf{h a}\right)$ \\
\hline CS & $28,094( \pm 7,8)$ \\
CC & $32,340( \pm 8,8)$ \\
PA & $28,246( \pm 6,6)$ \\
\hline
\end{tabular}

(superior a $18 \mathrm{~m}^{2} / \mathrm{ha}$ ), mas podem ser considerados baixos, pois valores maiores foram encontrados em outras FES, mesmo em áreas consideradas perturbadas, como é o caso de Dalanesi et al. (2004) (20,5 $\left.\mathrm{m}^{2} / \mathrm{ha}\right)$, Dan et al. (2010) $\left(48,81 \mathrm{~m}^{2} / \mathrm{ha} ; 28,35\right.$ $\mathrm{m}^{2} / \mathrm{ha} ; 27,30 \mathrm{~m}^{2} /$ ha e $25,89 \mathrm{~m}^{2} / \mathrm{ha}$ ), Archanjo et al. (2012) $\left(33,02 \mathrm{~m}^{2} / \mathrm{ha}\right)$ e França \& Stehmann (2013) $\left(36,41 \mathrm{~m}^{2} /\right.$ ha e $\left.39,48 \mathrm{~m}^{2} / \mathrm{ha}\right)$.

A área basal é um parâmetro diretamente proporcional ao estágio sucessional, e desse modo, esperávamos maior valor de área basal no sítio CS, por ser o sítio mais antigo, que não sofreu corte raso e pelo corte seletivo ter sido encerrado há 45 anos. O fato desse resultado não ter sido encontrado pode ser explicado pelos indícios da presença de pastoreio bovino no sítio CS, o que pode acarretar severos danos à estrutura vegetacional (Sampaio \& Guarino 2007). Outro fator relevante, é que a área basal é a variável mais prejudicada frente ao corte seletivo e os danos à estrutura podem permanecer por longos períodos (Gomes et al. 2004).

Quanto às distribuições diamétricas, os três sítios apresentaram uma distribuição comum às florestas inequiâneas, com um padrão exponencial em "J" reverso (Fig. 5). Constatamos que a densidade de indivíduos por classe de diâmetro é estatisticamente diferente entre os três sítios (Tab. 4). Ao comparar os sítios, o CS apresenta menor densidade de indivíduos, principalmente nas menores classes de diâmetro, contudo há indivíduos em todas as classes. Esse comportamento é comum em áreas que não sofreram corte raso como perturbação e demonstra maior desenvolvimento sucessional (Morel et al. 2016). Nos sítios CC e PA há maior proporção de indivíduos na maioria das classes de diâmetros, em relação ao sítio CS, mas há classes, a partir da classe de $30 \mathrm{~cm}$ de DAP, que apresentam-se interrompidas ou truncadas para os três sítios (Fig. 4), reforçando o histórico de perturbação que os sítios sofreram.

Em relação às lianas, foram registrados 2.283 indivíduos no fragmento florestal estudado, correspondendo a 3.003,95 ind/ha com área basal total de $2,43 \mathrm{~m}^{2} / \mathrm{ha}$ (Tab. 5). Os três sítios apresentaram indivíduos de lianas nas três classes de diâmetro, porém, a classe 3 (maior calibre), não apresentou diferença estatística entre os sítios. $\mathrm{O}$ sítio $\mathrm{CC}$ teve tendência de maior proporção de indivíduos na primeira classe de diâmetro (menor calibre) e o sítio PA destacou-se com o maior número de indivíduos na segunda classe de diâmetro.

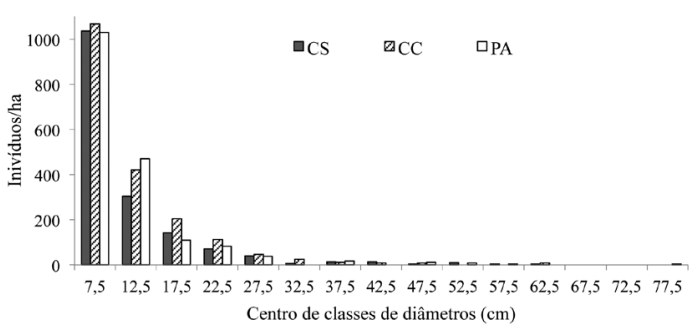

Figura 5 - Distribuição diamétrica dos indivíduos arbóreos amostrados nos sítios que compõem o Fragmento florestal, município de Alegre-ES. (CS = sítio de corte seletivo; $\mathrm{CC}=$ sítio de cultivo de café; $\mathrm{PA}=$ sítio de pastagens).

Figure 5 - Diametric distribution of the arboreal individuals sampled in the sites that make up the Forest fragment, Alegre-ES municipality. ( $\mathrm{CS}=$ selective cutting site; $\mathrm{CC}=$ coffee growing site; $\mathrm{PA}=$ pasture site). 
Tabela 4 - Lista dos parâmetros estatísticos gerados pelo teste de qui-quadrado $\left(\chi^{2}\right)$ para as distribuições diamétricas entre os sítios do fragmento florestal, município de Alegre-ES - CS = sítio de corte seletivo; CC = sítio de cultivo de café; PA = sítio de pastagens; G.L. = grau de liberdade.

Table 4 - List of statistical parameters generated by the chi-square test $\left(\chi^{2}\right)$ for the diametric distributions between the sites of the forest fragment, municipality of Alegre-ES - CS = selective cutting site; $C C=$ coffee growing site; PA = pasture site; G.L. = degree of freedom.

\begin{tabular}{lccc}
\hline Sítios & $\chi^{2}$ calc. & G.L. & Valor-p \\
\hline $\mathrm{CS} \times \mathrm{CC}$ & 83,9 & 11 & $<0,001$ \\
$\mathrm{CS} \times \mathrm{PA}$ & 198,4 & 11 & $<0,001$ \\
$\mathrm{CC} \times \mathrm{PA}$ & 200,0 & 11 & $<0,001$ \\
\hline
\end{tabular}

Tabela 5 - Parâmetros analisados para a comunidade de lianas amostradas em três glebas que compõem o Fragmento florestal, município de Alegre-ES - CS = sítio de corte seletivo; $\mathrm{CC}=$ sítio de cultivo de café; $\mathrm{PA}=$ sítio de pastagens. Valores encontrados ( \pm desvio padrão $)-\mathrm{AB} \mathrm{m}^{2} / \mathrm{ha}=$ área basal; $\mathrm{D}=$ densidade; Classe 1 = percentual de indivíduos na primeira classe de diâmetro $(1 \leq \mathrm{DAP}<2,5)$; Classe 2 = percentual de indivíduos na segunda classe de diâmetro $(2,5 \leq \mathrm{DAP}<5)$; Classe 3 = percentual de indivíduos na terceira classe de diâmetro (5 $\leq \mathrm{DAP})$. Os valores, quando não atenderam aos requisitos do teste de normalidade passaram por transformações $(*)$. Onde análises de variância indicaram diferenças significativas, médias seguidas por letras diferentes na coluna não diferiram significativamente entre si pelo teste de Tukey em $\mathrm{P} \leq 0,05$.

Table 5 - Parameters analyzed for the community of lianas sampled in three glebas that compose the Forest Fragment, Alegre-ES municipality $-\mathrm{CS}=$ selective cutting site $\mathrm{CC}=$ coffee growing site; $\mathrm{PA}=$ pasture site. Values found $( \pm$ standard deviation $)-\mathrm{AB} \mathrm{m}^{2} / \mathrm{ha}$ $=$ basal area; $\mathrm{D}=$ density; Classe $1=$ percentage of individuals in the first class of diameter $(1 \leq \mathrm{DAP}<2,5)$; Classe $2=$ percentage of individuals in the second class; Classe 3 = percentage of individuals in the third diameter class $(5 \leq \mathrm{DAP})$. The values, when they did not meet the normality test requirements, underwent transformations $(*)$. Where analyzes of variance indicated significant differences, mean values followed by different letters in the column did not differ significantly from each other by the Tukey test at $\mathrm{P} \leq 0.05$.

\begin{tabular}{cccccc}
\hline \multicolumn{7}{c}{ Lianas no Fragmento Florestal } \\
\hline Sítio & $\mathbf{D}$ (ind/ha) & $\mathbf{A B}\left(\mathbf{m}^{\mathbf{2}} / \mathbf{h a}\right) *$ & Classe 1 (\%) & Classe 2 (\%) & Classe 3 (\%)* \\
\hline CS & $3828( \pm 2309)$ & $2,667( \pm 1,5)$ & $55( \pm 15,19) \mathrm{ab}$ & $39( \pm 13,72) \mathrm{ab}$ & $6( \pm 9,67)$ \\
$\mathrm{CC}$ & $2383( \pm 1397)$ & $1,258( \pm 0,5)$ & $69( \pm 17,23) \mathrm{a}$ & $28( \pm 14,94) \mathrm{b}$ & $3( \pm 10,02)$ \\
$\mathrm{PA}$ & $2101( \pm 2101)$ & $3,369( \pm 2,8)$ & $44( \pm 15,16) \mathrm{b}$ & $44( \pm 15,06) \mathrm{a}$ & $12( \pm 8,93)$ \\
\hline
\end{tabular}

A maior abundância de lianas de pequeno diâmetro segue o padrão de florestas tropicais perturbadas (Carvalho et al. 2011). Menor densidade e maiores diâmetros de lianas podem ser um indicativo de florestas em estágios sucessionais mais avançados (Laurance et al. 2001), conforme encontrado por Letcher \& Chazdon (2009). Esses autores compararam a densidade e diâmetros de lianas em cinco florestas com diferentes tempos de pousio e verificaram que o comprimento do diâmetro é proporcional à idade da floresta, contudo a densidade de caules diminuíram. Isso pode ocorrer devido á características que são alteradas com o avanço da sucessão florestal, como por exemplo a menor penetração de luz abaixo do dossel (Letcher \& Chazdon 2009). Nesse sentido, na presente pesquisa, acreditamos que o efeito de borda está influenciando na maior densidade de lianas de pequeno diâmetro nos sítios CS e CC (Laurance et al. 2001). O sítio PA possui posicionamento no fragmento que lhe proporciona menor borda, pode ser por isso que encontramos menor proporção de lianas de menor diâmetro (Letcher \& Chazdon 2009; Carvalho et al. 2011).

Em função do exposto, acreditamos o efeito de borda pode estar influenciando na recuperação dos sítios CS e PA. Estes resultados podem direcionar medidas conservacionistas que contribuem para minimizar os efeitos de borda para FES com históricos de perturbações semelhantes, visto que, pastagens e cultivo de café são comuns na região estudada. Além disso, é relevante adotar medidas que 
minimizem as perturbações no fragmento florestal, como evitar o pastoreio bovino.

Os resultados obtidos na presente pesquisa contribuem para o conhecimento da flora regional, principalmente para o sul do estado do Espírito Santo, onde são escassos os estudos florísticos e fitossociológicos. Além disso, o fragmento florestal estudado possui grande importância na conservação da biodiversidade local e na realização de educação ambiental por meio das trilhas ecológicas nele implantadas, que promovem o conhecimento sobre o bioma Mata Atlântica na região.

\section{Considerações finais}

A análise da categoria sucessional demonstrou que os sítios CS e PA possuem estágio mais avançado de sucessão em relação à $\mathrm{CC}$. As análises relacionadas às espécies de maior VI, Às síndromes de dispersão e às lianas apontaram estágio sucessional mais avançado somente no sítio PA. As demais análises não demonstraram diferença entre os três sítios, refutando as hipóteses. Acreditamos que o maior avanço no estágio sucessional do sítio PA deve-se à sua localização no centro do fragmento florestal, maior proteção aos efeitos de borda e aos efeitos antrópicos por estar dentro dos limites do PEAMA e contar com fiscalização.

Por meio da presente pesquisa podemos dizer que o tempo de pousio dos sítios proporcionou uma equivalência nos estágios sucessionais dos sítios que compõem o fragmento florestal estudado e que, o interior do fragmento possui características de estágios mais avançados de sucessão, independentemente do tipo de uso anterior do solo.

\section{Agradecimentos}

O presente trabalho foi realizado com o apoio da Fundação de Amparo à Pesquisa e Inovação do Espírito Santo (FAPES) / Coordenação de Aperfeiçoamento de Pessoal de Nível Superior Brasil (CAPES) - Edital FAPES/CAPES No 02/2014 - bolsa de Mestrado.

\section{Referências}

Abreu KMP, Silva GF \& Silva AG (2013) Análise fitossociológica da Floresta Nacional de Pacotuba, Cachoeiro de Itapemirim, ES-Brasil. Cerne 19: 157-168.

Archanjo KMPA, Silva GF, Chichorro JF \& Soares CPB (2012) Estrutura do componente arbóreo da Reserva Particular do Patrimônio Natural Cafundó, Cachoeiro de Itapemirim, Espírito Santo, Brasil. Floresta 42: 145-160.
Arruda DM, Ferreira-Junior WG, Duque-Brasil R \& Schaefer CE (2013) Phytogeographical patterns of dry forests sensu stricto in northern Minas Gerais state, Brazil. Anais da Academia Brasileira de Ciências 85: 623-634.

Braga AJT, de Lima EE \& Martins SV (2011) Florística e estrutura da comunidade arbórea de uma Floresta Estacional Semidecidual secundária em Viçosa, MG. Árvore 35: 493-503.

Braga AJT, Lima EE, Borges VM \& Martins SV (2015) Influência dos fatores edáficos na variação florística de Floresta Estacional Semidecidual, em Viçosa, MG. Árvore 39: 623-633.

Carvalho FA (2010) Síndromes de dispersão de espécies arbóreas de Florestas Ombrófilas Submontanas do estado do Rio de Janeiro. Árvore 34: 1017-1023.

Carvalho FA \& Nascimento MT (2009) Estrutura diamétrica da comunidade e das principais populações arbóreas de um remanescente de Floresta Atlântica Submontana (Silva Jardim-RJ, Brasil). Revista Árvore 33: 327-337.

Carvalho FA, Braga JMA, Gomes JML, Souza JS \& Nascimento MT (2006) Comunidade arbórea de uma floresta de baixada aluvial no município de Campos dos Goytacazes, RJ. Cerne 12: 157-166.

Carvalho PG, Mellis JV, Ascenção BM, Cestari FM, Alves LF \& Grombone-Guaratini MT (2011) Abundância e biomassa de lianas em um fragmento de floresta Atlântica. Hoehnea 38: 307-314.

Carvalho WAC, Oliveira Filho AT, Fontes MAL \& Curi N (2007) Variação espacial da estrutura da comunidade arbórea de um fragmento de floresta semidecídua em Piedade do Rio Grande, MG, Brasil. Revista Brasileira de Botânica 30: 315-335.

CIENTEC (2010) Software Mata Nativa 2.06: sistema para análise fitossociológica, elaboração de inventários e planos de manejo de florestas nativas. Viçosa, MG. Disponível em $<$ https://www. matanativa.com.br>. Acesso em novembro 2015.

CONAMA (1994) Resolução n ${ }^{\circ}$ 29, de 7 de dezembro de 1994. Define vegetação primária e secundária nos estágios inicial, médio e avançado de regeneração da Mata Atlântica, considerando a necessidade de definir o corte, a exploração e a supressão de vegetação secundária no estágio inicial de regeneração no estado do Espírito Santo. Diário Oficial da União 248: 21349-21350.

Dalanesi PE, Oliveira-Filho AT \& Fontes MAL (2004) Flora e estrutura do componente arbóreo da floresta do Parque Ecológico Quedas do Rio Bonito, LavrasMG, e correlações entre distribuição das espécies e variáveis ambientais. Acta Botanica Brasilica 18: 737-757.

Dan ML, Braga JMA \& Nascimento MT (2010) Estrutura da comunidade arbórea de fragmentos de floresta estacional semidecidual na bacia hidrográfica do rio São Domingos, Rio de Janeiro, Brasil. Rodriguésia 61: 749-766. 
D’Antonio CM \& Vitousek PM (1992) Biological invasions by exotics grasses, the grass/fire cycle, and global change. Annual Review of Ecology and Systematics 63: 63-87.

Durigan G, Bernacci LC, Franco GADC, Arbocz GF, Metzger JP \& Catharino ELM (2008) Estádio sucessional e fatores geográficos como determinantes da similaridade florística entre comunidades florestais no Planalto Atlântico, estado de São Paulo, Brasil. Acta Botanica Brasilica 22: 51-62.

EMBRAPA - Empresa brasileira de pesquisa agropecuária (2006) Sistema brasileiro de classificação de solos. $2^{\mathrm{a}}$ ed. Embrapa Solos, Rio de Janeiro. 306p.

Estevan DA, Vieira AOS \& Gorenstein MR (2016) Estrutura e relações florísticas de um fragmento de Floresta Estacional Semidecidual, Londrina, Paraná, Brasil. Ciência Florestal 26: 713-725.

Forzza RC, Baumgratz JFA, Bicudo CEM, Canhos DAL, Carvalho Junior AA, Coelho MAN, Costa AF, Costa DP, Hopkins MG, Leitman PM, Lohmann LG, Lughadha EM, Maia LC, Martinelli G, Menezes M, Morim MP, Peixoto AL, Pirani JR, Prado J, Queiroz LP, Souza S, Souza VC, Stehmann JR, Sylvestre LS, Walter BMT \& Zappi DC (2012) New brazilian floristic list highlights conservation challenges. Bioscience 62: 39-45.

Franco BKS, Martins SV, Faria PCL, Ribeiro GA \& Neto AM (2014) Estrato de regeneração natural de um trecho de Floresta Estacional Semidecidual, Viçosa, MG. Árvore 38: 31-40.

França GS \& Stehmann JR (2013) Florística e estrutura do componente arbóreo de remanescentes de Mata Atlântica do médio rio Doce, Minas Gerais, Brasil. Rodriguésia 64: 607-624.

Fundação SOS Mata Atlântica \& Instituto Nacional de Pesquisas Espaciais - INPE (2015) Atlas dos remanescentes florestais da Mata Atlântica 20132014, São Paulo. Disponível em < https://www. sosma.org.br/14622/divulgados-novos-dadossobre-a-situacao-da-mata-atlantica/>. Acesso em junho 2017.

Gandolfi S, Leitão Filho HF \& Bezerra CLF (1995) Levantamento florístico e caráter sucessional das espécies arbustivo-arbóreas de uma Floresta Semidecídua no município de Guarulhos, SP. Revista Brasileira de Botânica 55: 753-767.

Gomes APC, Souza AL \& Meira Neto JAA (2004) Alteração estrutural de uma área florestal explorada convencionalmente na Bacia do Paraíba do Sul, Minas Gerais, nos domínios de Floresta Atlântica. Árvore 28: 407-417.

Gotelli NJ \& Entsminger GL (2011) EcoSim: null models software for ecology. Version 7.0. Acquired Intelligence Inc. \& Kesey-Bear. Jericho, VT 05465. Disponível em $<$ http:/garyentsminger.com/ecosim/ index.htm $>$. Acesso em agosto 2017.

Guedes RS, Zanella FCV, Costa Junior JEV, Santana GM \& Silva JA (2012) Caracterização florístico- fitossociológica do componente lenhoso de um trecho de caatinga no semiárido paraibano. Revista Caatinga 25: 99-108.

Gusson AE, Lopes SF, Dias Neto OC, Vale VS, Oliveira AP \& Schiavini I (2009) Características químicas do solo e estrutura de um fragmento de Floresta Estacional Semidecidual em Ipiaçu, Minas Gerais, Brasil. Rodriguésia 60: 403-414.

IEMA - Instituto Estadual de Meio Ambiente e Recursos Hídricos (2015) Lista da flora ameaçada de extinção. Disponível em <http://www.meioambiente.es.gov. br/web/flora.htm>. Acesso em 26 outubro 2015.

Kent M \& Coker P (1992) Vegetation description and analysis: a practical approach. John Wiley \& Sons, New York. 363p.

Kovach WL (2004) MVSP (Multivariate Statistical Package), version $3.13 \mathrm{~m}$. Kovach Computing Service, Wales. Disponível em <https://www. kovcomp.co.uk/kcssoft.html $>$. Acesso em dezembro 2015.

Köppen W (1948) Climatologia: con un estudio de los climas de la tierra. Fondo de Cultura Econômica, Ciudad de México. 479p.

Laurance WF, Pérez-Salicrup D, Delamônica P, Fearnside PM, D'angelo S, Jerozolinski A, Pohl L \& Lovejoy TE (2001) Rain forest fragmentation and the structure of Amazonian liana communities. Ecology 82: 105-116.

Letcher SG \& Chazdon RL (2009) Lianas and self-supporting plants during tropical forest succession. Forest Ecology and Management 257: 2150-2156.

Lima JSS, Silva SA, Oliveira RB, Cecílio RA \& Xavier AC (2008) Variabilidade temporal da precipitação mensal em Alegre-ES. Revista Ciência Agronômica 39: 327-332.

Lira PK, Tambosi LR, Ewers RM \& Metzger JP (2012) Land-use and land-cover change in Atlantic Forest landscapes. Forest Ecology and Management 278: 80-89.

Lopes WP, Silva AF, Souza AL \& Meira Neto JAA(2002) Estrutura fitossociológica de um trecho de vegetação arbórea no Parque Estadual do Rio Doce - Minas Gerais, Brasil. Acta Botânica Brasílica 16: 443-456.

López-Gómez AM, Williams-Linera G \& Manson RH (2008) Tree species diversity and vegetation structure in shade coffee farms in Veracruz, Mexico. Agriculture, ecosystems \& environment 124: $160-172$.

Lugo AE \& Helmer E (2004) Emerging forests on abandoned land: Puerto Rico's new forests. Forest Ecology and Management 190: 145-161.

Magurran A (1988) Ecological Diversity and its measurement. University Press, Cambridge. 179p.

Magurran AE (2004) Measuring biological diversity. Blackwell Science, Oxford. 256p.

Marques M, Zwiener VP, Ramos FM, Borgo M \& Marques R (2014) Forest structure and species 
composition along a successional gradient of Lowland Atlantic Forest in Southern Brazil. Biota Neotropica 14: 1-11.

Myers N, Mittermeier RA, Mittermeier CG, Da Fonseca GA \& Kent J (2000) Biodiversity hotspots for conservation priorities. Nature 403: 853-858.

Morel JD, Pereira JAA, Santos RM, Machado ELM \& Marques JJ (2016) Diferenciação da vegetação arbórea de três setores de um remanescente florestal relacionada ao seu histórico de perturbações. Ciência Florestal 26: 81-93.

Mota TJ, Carvalho FA, Ivanauskas NM \& Eisenlohr PV (2017) On the relevance of floristic and quantitative studies to the restoration of degraded areas: the case of the Atlantic Forest hotspot. Aims Environmental Science 4: 42-53.

Martinelli G \& Moraes MA (2013) Livro Vermelho da Flora do Brasil. Instituto de Pesquisas Jardim Botânico do Rio de Janeiro \& Andrea Jakobson, Rio de Janeiro. 1100p.

Moreira LN, Moreno MR \& Redling JSH (2007) Estrutura populacional de Senefeldera multiflora em um trecho de borda na Mata Atlântica Estacional Semidecidual da Floresta Nacional de Pacotuba, Cachoeiro de Itapemirim, Espírito Santo. Revista Brasileira de Biociências 5: 669-671.

Mueller-Dombois D \& Ellenberg H (2002) Aims and methods of vegetation ecology. The Blackburn Press, New Jersey. 547p.

Pezzopane JE, Oliveira PD, Reis ED \& Lima JDS (2004) Alterações microclimáticas causadas pelo uso de tela plástica. Engenharia Agrícola 24: 9-15.

Ribeiro RD \& Lima HC (2009) Riqueza e distribuição geográfica de espécies arbóreas da família Leguminosae e implicações para conservação no centro de diversidade vegetal de Cabo Frio, Rio de Janeiro, Brasil. Rodriguésia 60: 111-127.

Sampaio MB \& Guarino ESG (2007) Efeitos do pastoreio de bovinos na estrutura populacional de plantas em fragmentos de Floresta Ombrófila Mista. Árvore 31: 1035-1046.

Silva AF, Oliveira RV, Santos NRL \& Paula A (2003) Composição florística e grupos ecológicos das espécies de um trecho de Floresta Semidecídua Submontana da fazenda São Geraldo, Viçosa-MG. Árvore 27: 311-319.

Silva FAS \& Azevedo CAV (2009) Principal componentes analysis in the software Assistat-Statistical Attendance. In: World congress on computers in agriculture. Vol. 7. American Society of Agricultural and Biological Engineers, Reno. Pp. 393-396.

Silva WM, Zorzanelli JPF, Moreau JS, Abreu KMP, Kunz SH (2017) Estrutura e sucessão ecológica de uma comunidade florestal urbana no sul do Espírito Santo. Rodriguésia 67: 301-314.

Souza JS, Espírito-Santo FDB, Fontes MAL, OliveiraFilho AT \& Botezelli L (2003) Análise das variações florísticas e estruturais da comunidade arbórea de um fragmento de Floresta Semidecídua às margens do Rio Capivari, Lavras-MG. Árvore 27: 185-206.

Rabelo GR, Klein DE \& Cunha M (2012) Does selective logging affect the leaf structure of a late successional species? Rodriguésia 63: 419-427.

Oliveira-Neto NE, Nascimento DR \& Carvalho FA(2017) Biodiversity inventory of trees in a neotropical secondary forest after abandonment of shaded coffee plantation. iForest-Biogeosciences and Forestry 10: 303-308.

Tabarelli M, Aguiar AV, Ribeiro MC, Metzger JP \& Peres CA (2010) Prospects for biodiversity conservation in the Atlantic Forest: lessons from aging humanmodified landscapes 143: 2328-2340.

Tabarelli M, Peres CA\& Melo FP(2012) The "few winners and many losers" paradigm revisited: emerging prospects for tropical forest biodiversity. Biological Conservation 155: 136-140.

IUCN (2016) The IUCN Red List of Threatened Species. Version 2016-3. Disponível em <http://www. iucnredlist.org > . Acesso em 3 março 2017.

Van der Pijl L (1982) Principles of dispersal in higher plants. $3^{\text {rd }}$ ed. Springer-Verlag, Berlin. 161p.

Veloso HP, Rangel Filho ALR \& Lima JCA (1991) Classificação da vegetação brasileira, adaptada a um sistema universal. IBGE, Rio de Janeiro. 124p.

Zar JH (1999) Biostatistical analysis. Prentice Hall, Upper Saddle River. Pp. 231-272.

Editor de área: Dr. Anderson Alves-Araújo

Artigo recebido em 09/07/2017. Aceito para publicação em 29/12/2017. 